\title{
Unexpected metabolic versatility in a combined fungal fomannoxin/vibralactone biosynthesis
}

Daniel Schwenk,,$^{\dagger}$ Philip Brandt,,$^{\dagger}$ Robert A. Blanchette,${ }^{\dagger}$ Markus Nett, ${ }^{\S}{ }^{\perp}$ Dirk Hoffmeister ${ }^{\dagger},{ }^{*}$

${ }^{\dagger}$ Department of Pharmaceutical Microbiology at the Hans-Knöll-Institute, Friedrich-SchillerUniversität, Beutenbergstrasse 11a, 07745 Jena, Germany

University of Minnesota, Plant Pathology, 1991 Upper Buford Circle, Saint Paul, MN 55108, USA

${ }^{\S}$ Leibniz Institute for Natural Product Research and Infection Biology, Hans-Knöll-Institute, Beutenbergstrasse 11a, 07745 Jena, Germany

${ }^{\perp}$ Department of Biochemical and Chemical Engineering, Technical Biology, Technical University Dortmund, Emil-Figge-Strasse 66, 44227 Dortmund, Germany

\section{AUTHOR INFORMATION}

\section{Corresponding Author}

*Phone: +49 3641 949850, Fax: +49 3641 949852, E-mail: dirk.hoffmeister@hki-jena.de 


\section{Table of contents}

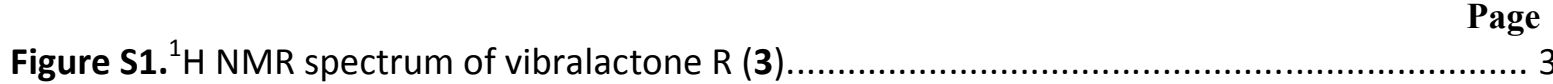

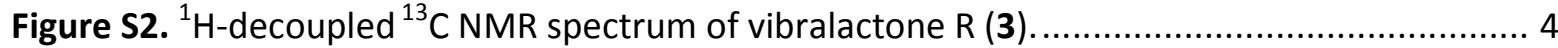

Figure S3. ${ }^{1} \mathrm{H}$-decoupled ${ }^{13} \mathrm{C}$ NMR spectrum of 3, isolated from $\left[1-{ }^{13} \mathrm{C}\right]$ acetate-supplemented

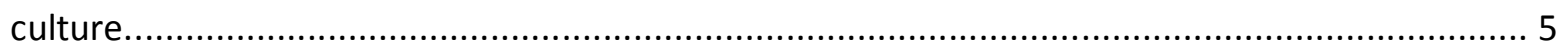

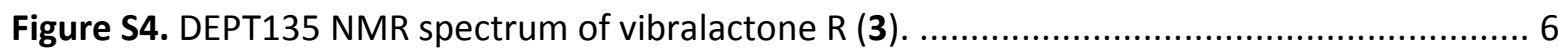

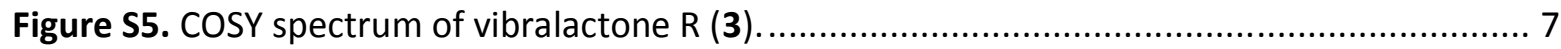

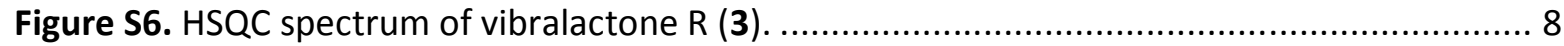

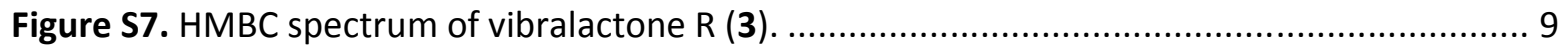

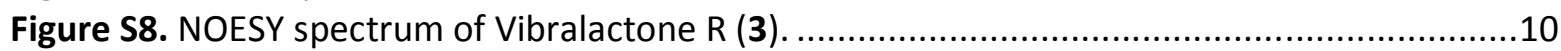

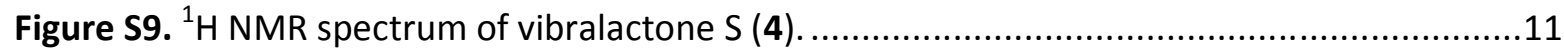

Figure S10. ${ }^{1} \mathrm{H}$-decoupled ${ }^{13} \mathrm{C}$ NMR spectrum of vibralactone $\mathrm{S}(4)$.......................................12

Figure S11. ${ }^{1} \mathrm{H}$-decoupled ${ }^{13} \mathrm{C}$ NMR spectrum of 4 , isolated from $\left[1-{ }^{13} \mathrm{C}\right]$ acetate-supplemented

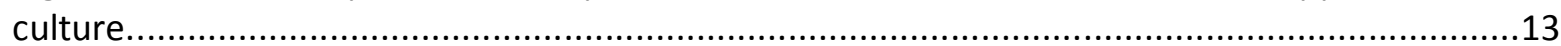

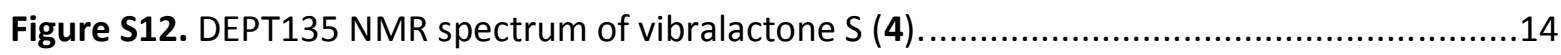

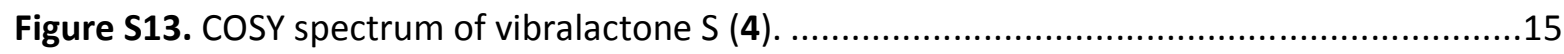

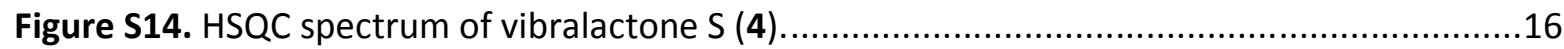

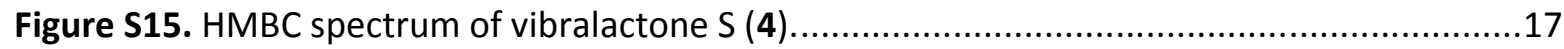

Figure S16. ${ }^{1} \mathrm{H}$ NMR spectrum of methyl seco-fomannoxinate (6). ............................................18

Figure S17. ${ }^{1} \mathrm{H}$-decoupled ${ }^{13} \mathrm{C}$ NMR spectrum of methyl seco-fomannoxinate (6).......................19

Figure S18. ${ }^{1} \mathrm{H}$-decoupled ${ }^{13} \mathrm{C}$ NMR spectrum of 6, isolated from $\left[1-{ }^{13} \mathrm{C}\right]$ acetate-supplemented

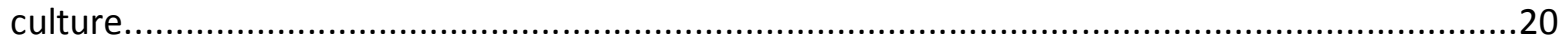

Figure S19. DEPT135 pectrum of methyl seco-fomannoxinate (6). .......................................21

Figure S20. COSY spectrum of methyl seco-fomannoxinate (6)..........................................22

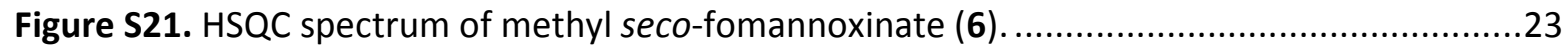

Figure S22. HMBC spectrum of methyl seco-fomannoxinate (6)........................................24

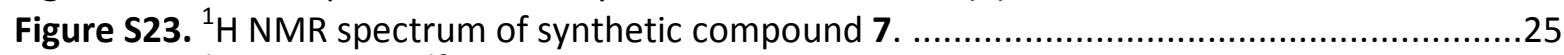

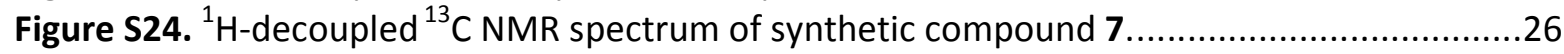

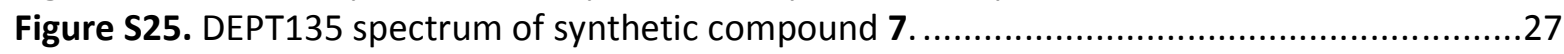

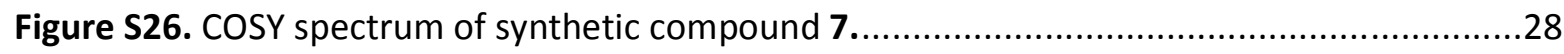

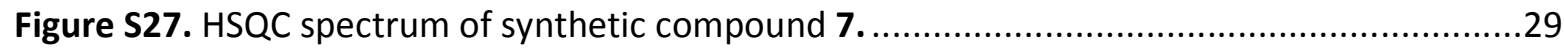

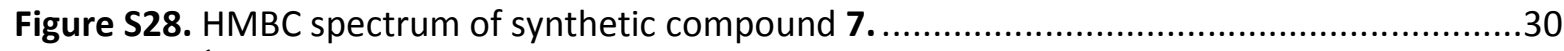

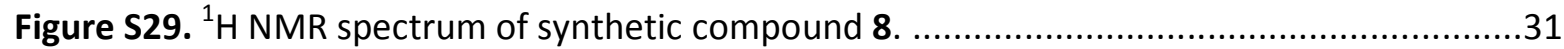

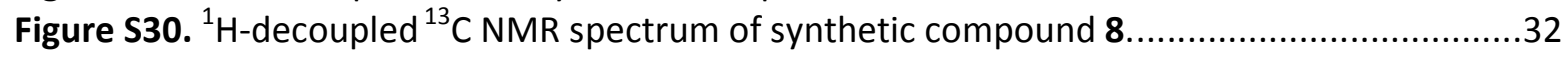

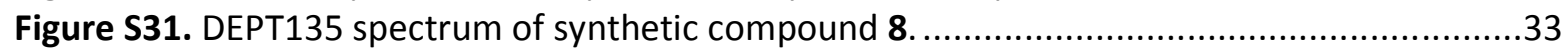

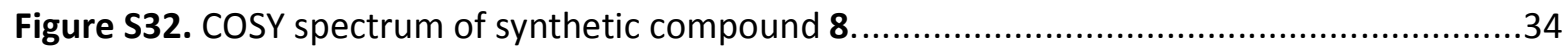

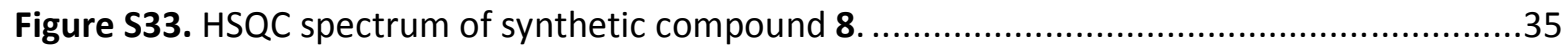

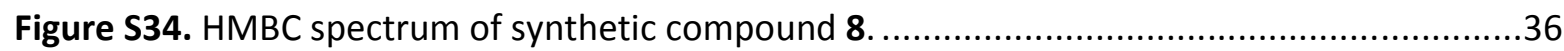

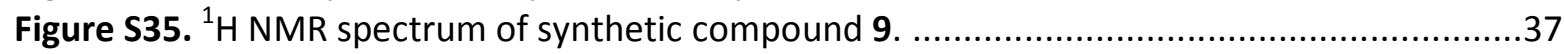

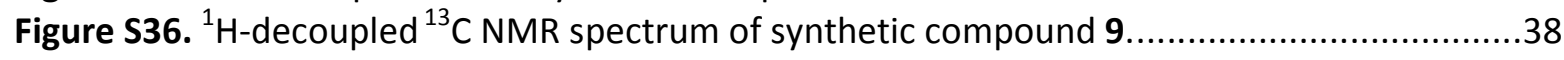

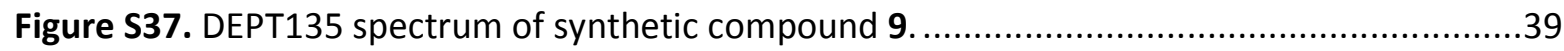

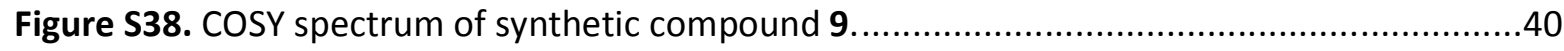

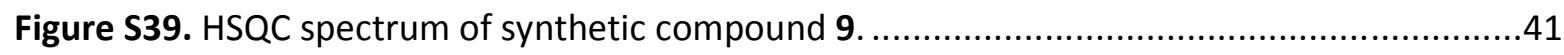

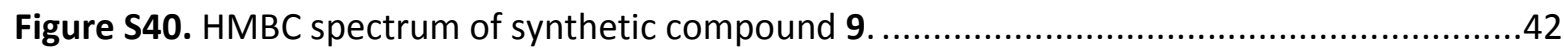




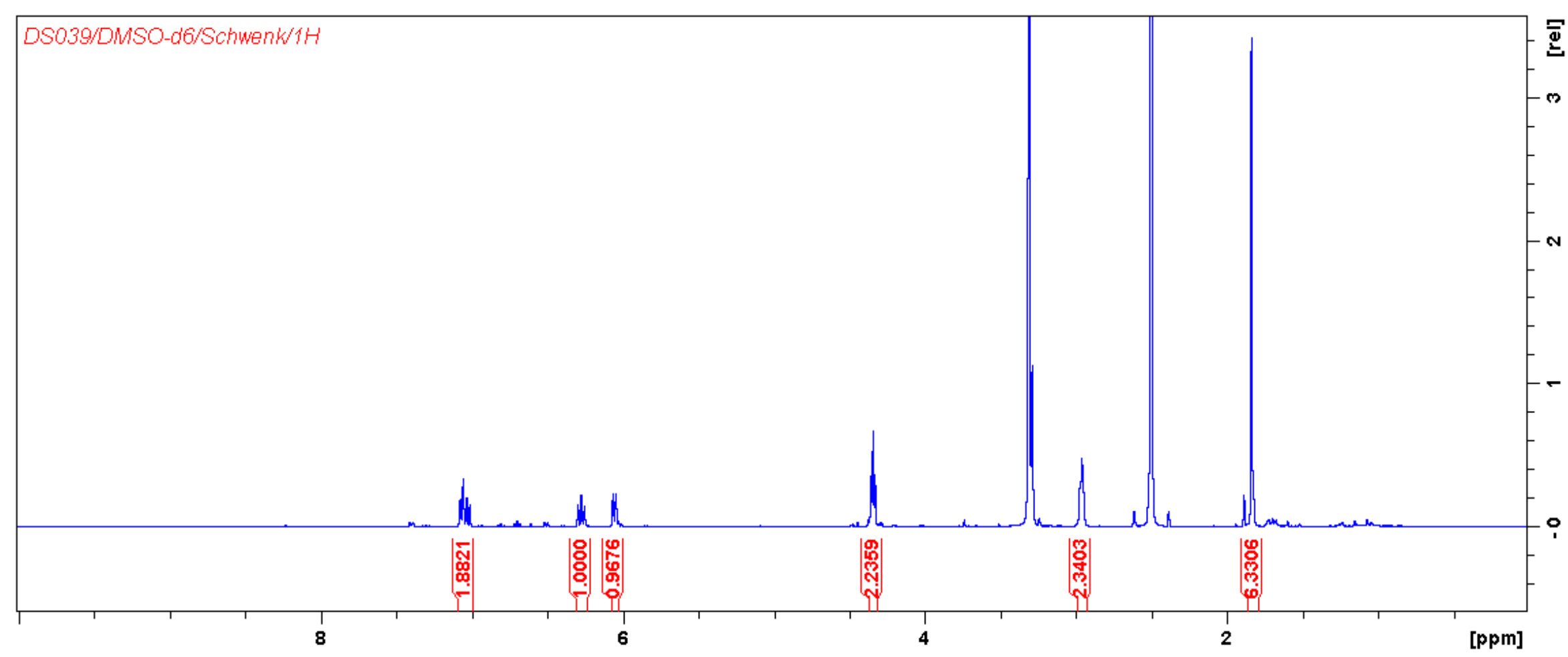

Figure S1. ${ }^{1} \mathrm{H}$ NMR spectrum of vibralactone $\mathrm{R}(\mathbf{3})$. 


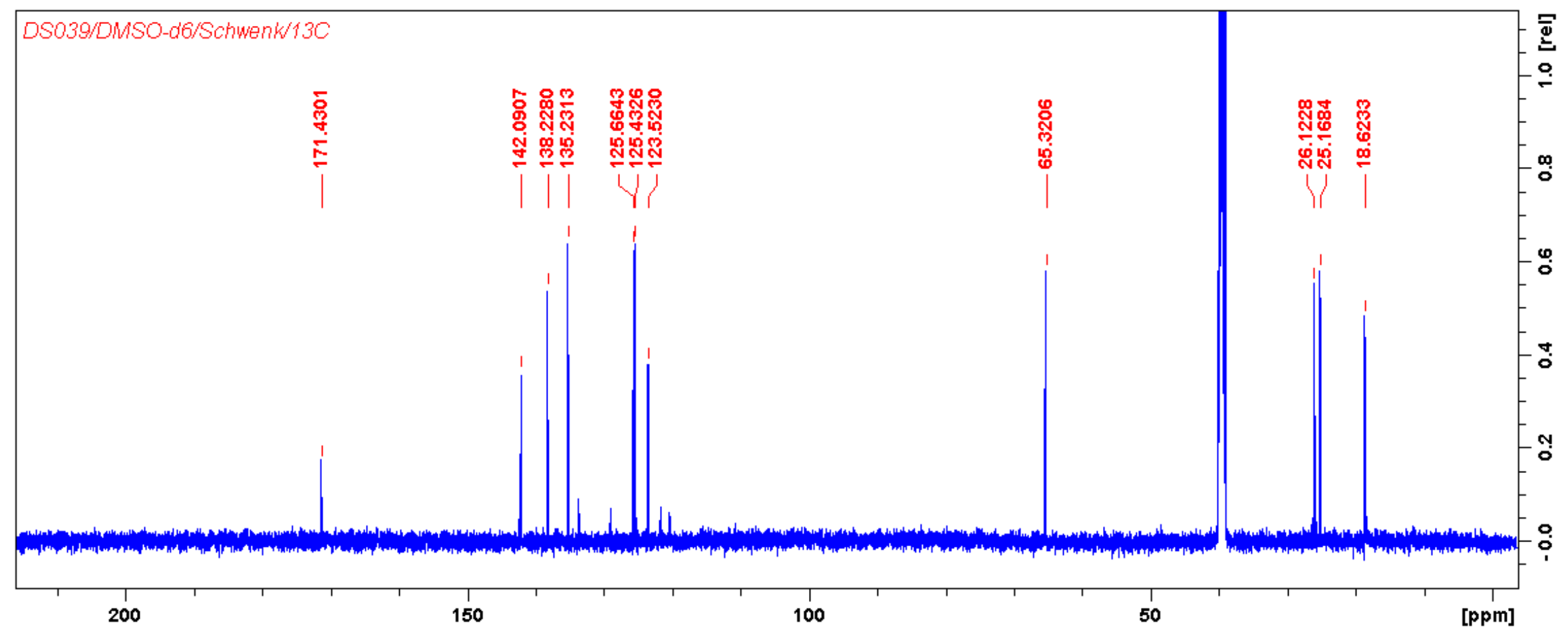

Figure S2. ${ }^{1} \mathrm{H}$-decoupled ${ }^{13} \mathrm{C}$ NMR spectrum of vibralactone $\mathrm{R}(\mathbf{3})$. 


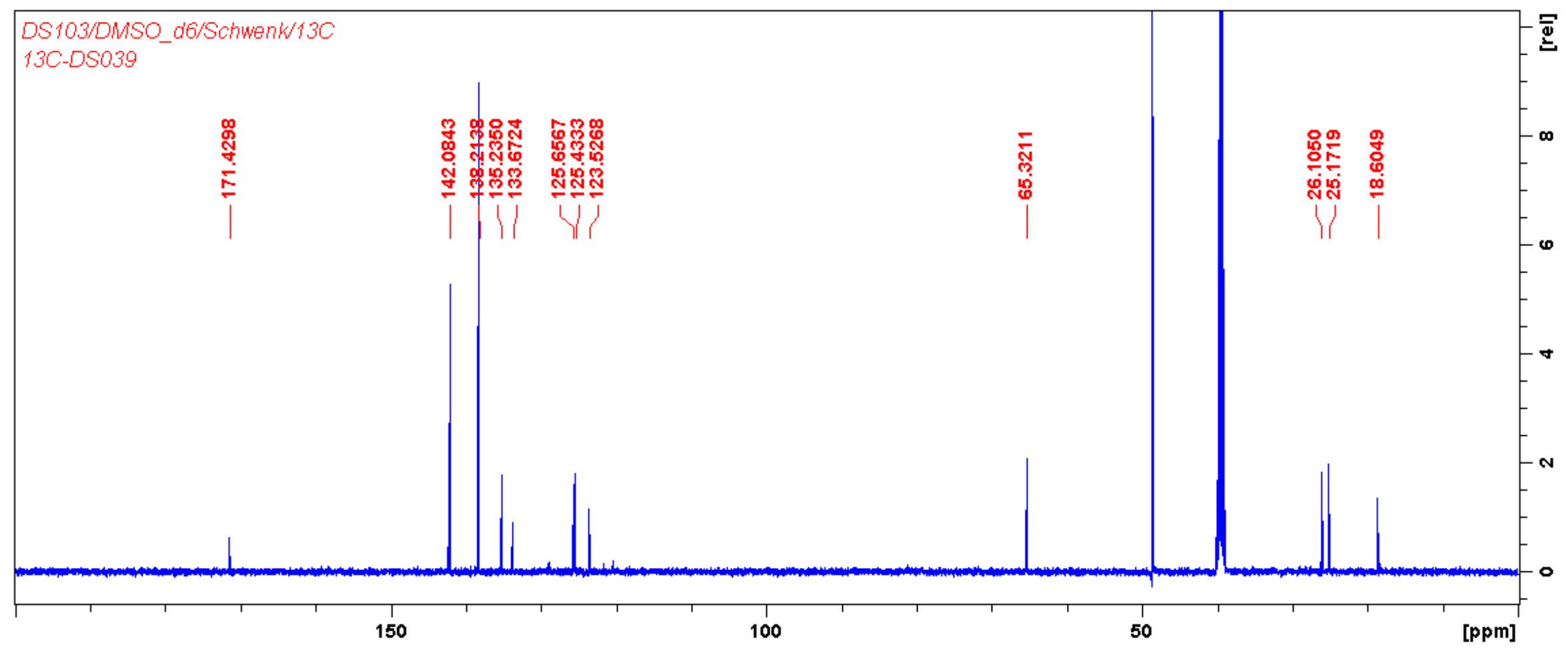

Figure S3. ${ }^{1} \mathrm{H}$-decoupled ${ }^{13} \mathrm{C}$ NMR spectrum of 3, isolated from $\left[1-{ }^{13} \mathrm{C}\right]$ acetate-supplemented culture. 


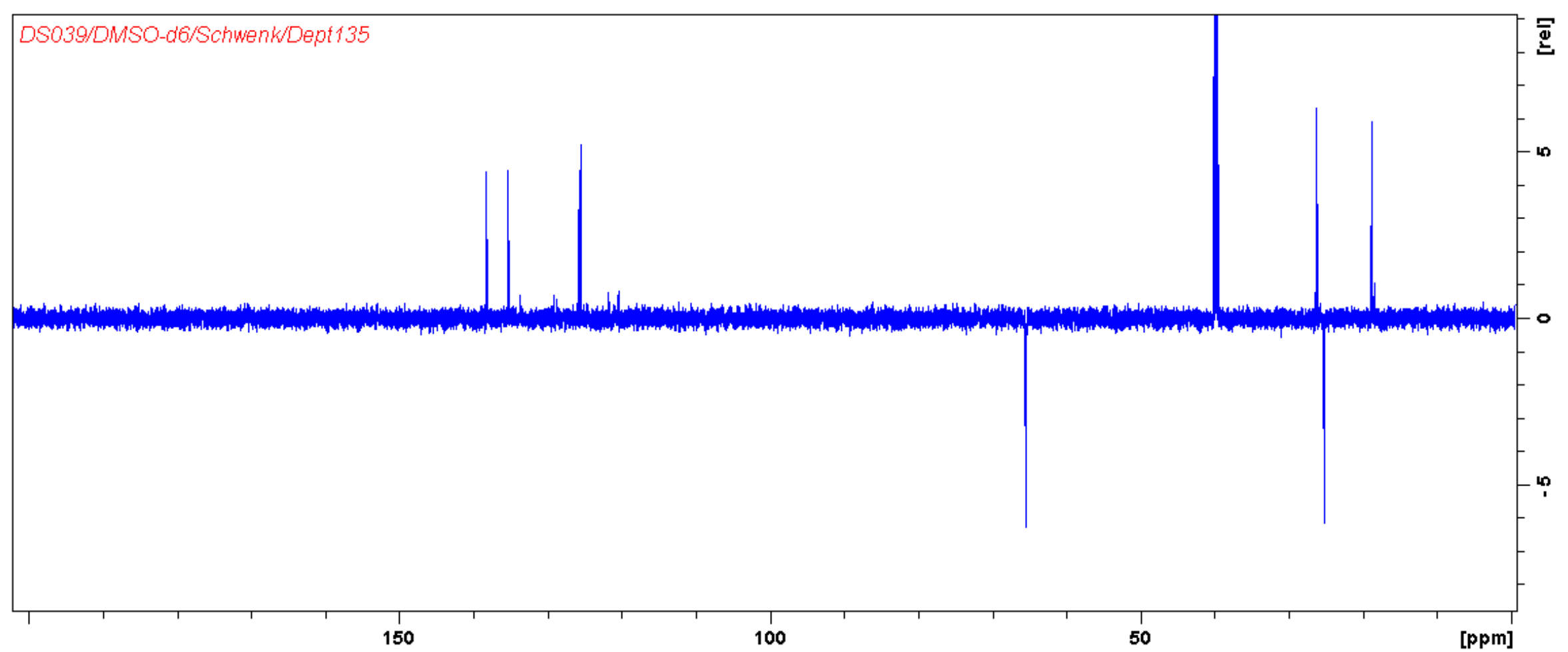

Figure S4. DEPT135 NMR spectrum of vibralactone R (3). 


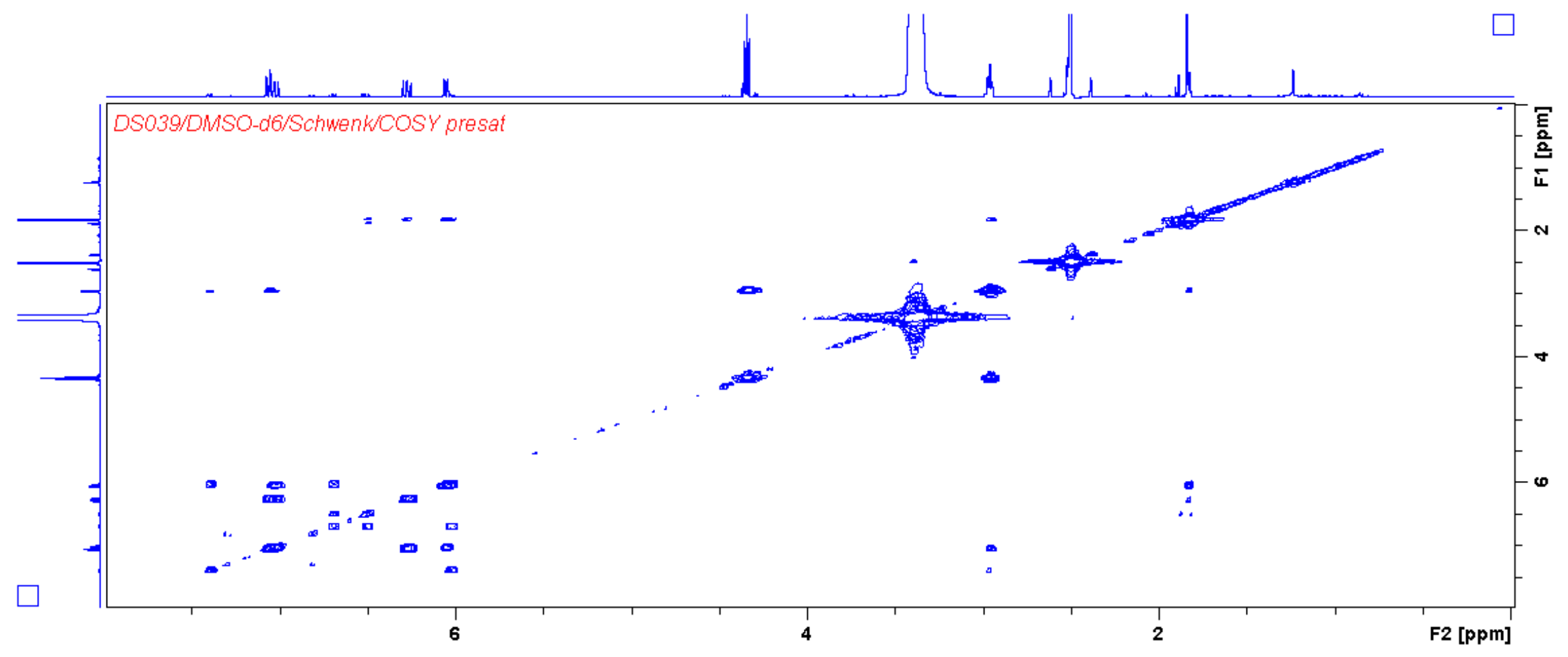

Figure S5. COSY spectrum of vibralactone R (3). 


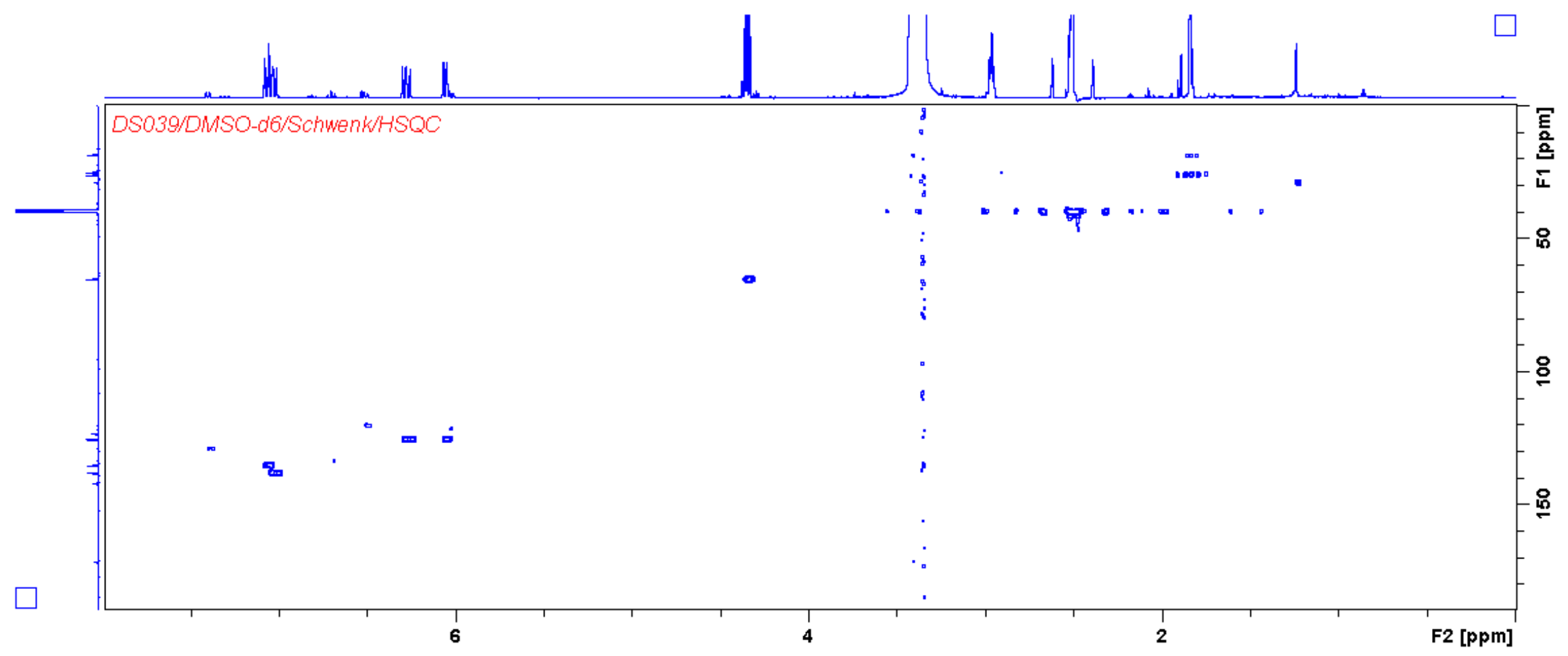

Figure S6. HSQC spectrum of vibralactone R (3). 


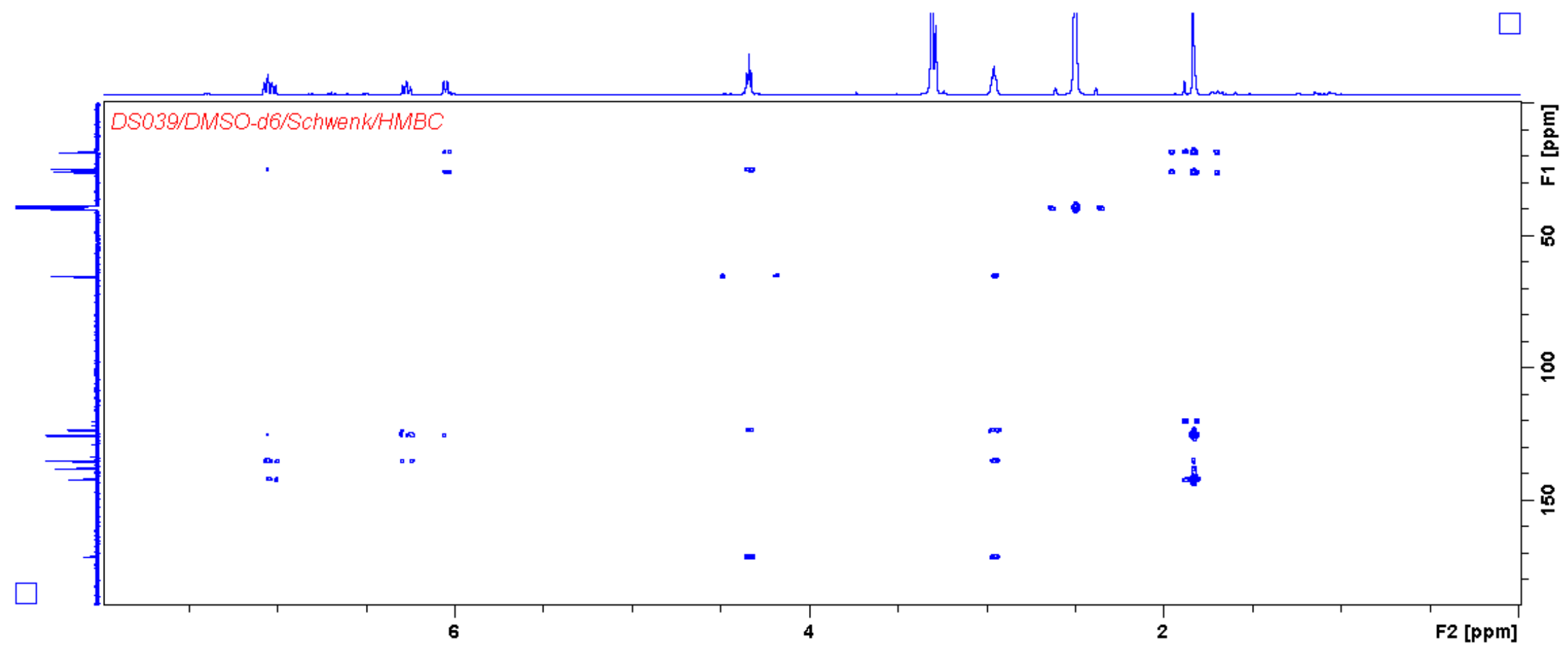

Figure S7. HMBC spectrum of vibralactone R (3). 


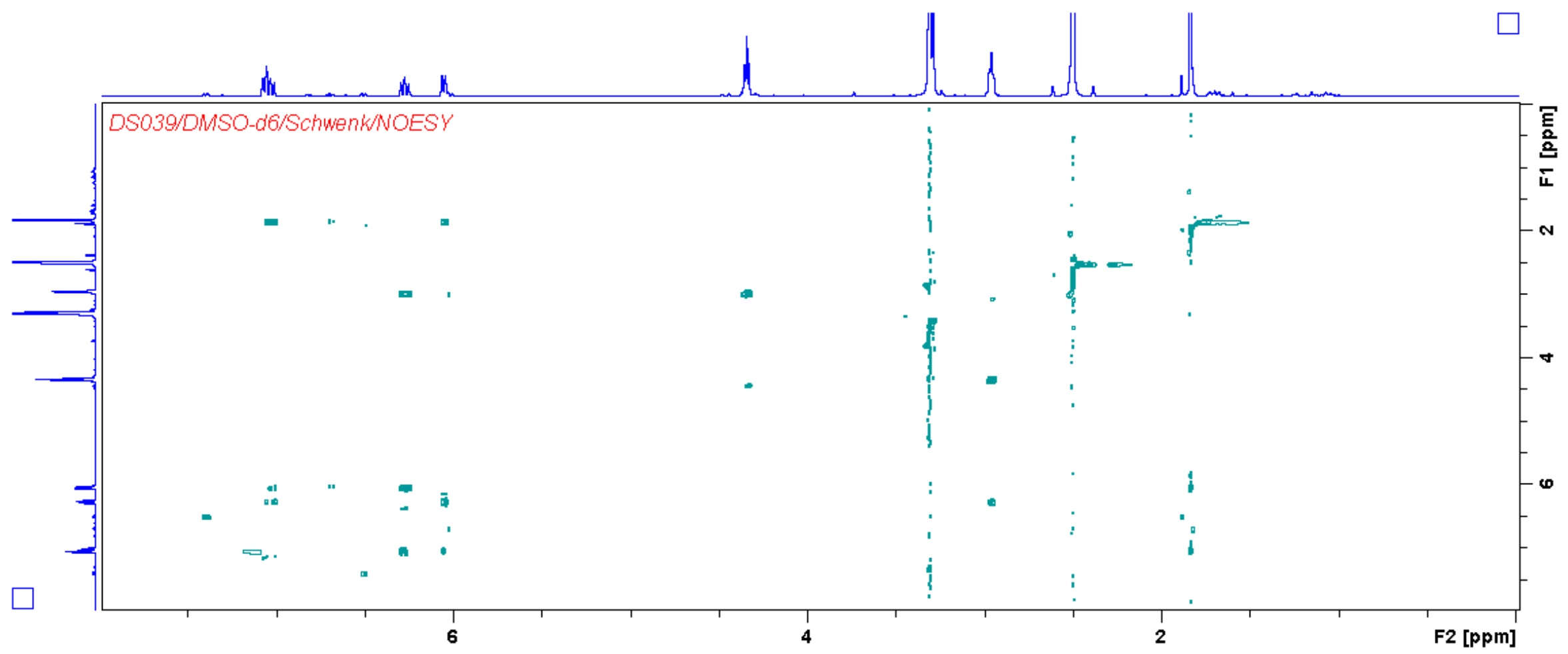

Figure S8. NOESY spectrum of Vibralactone R (3). 


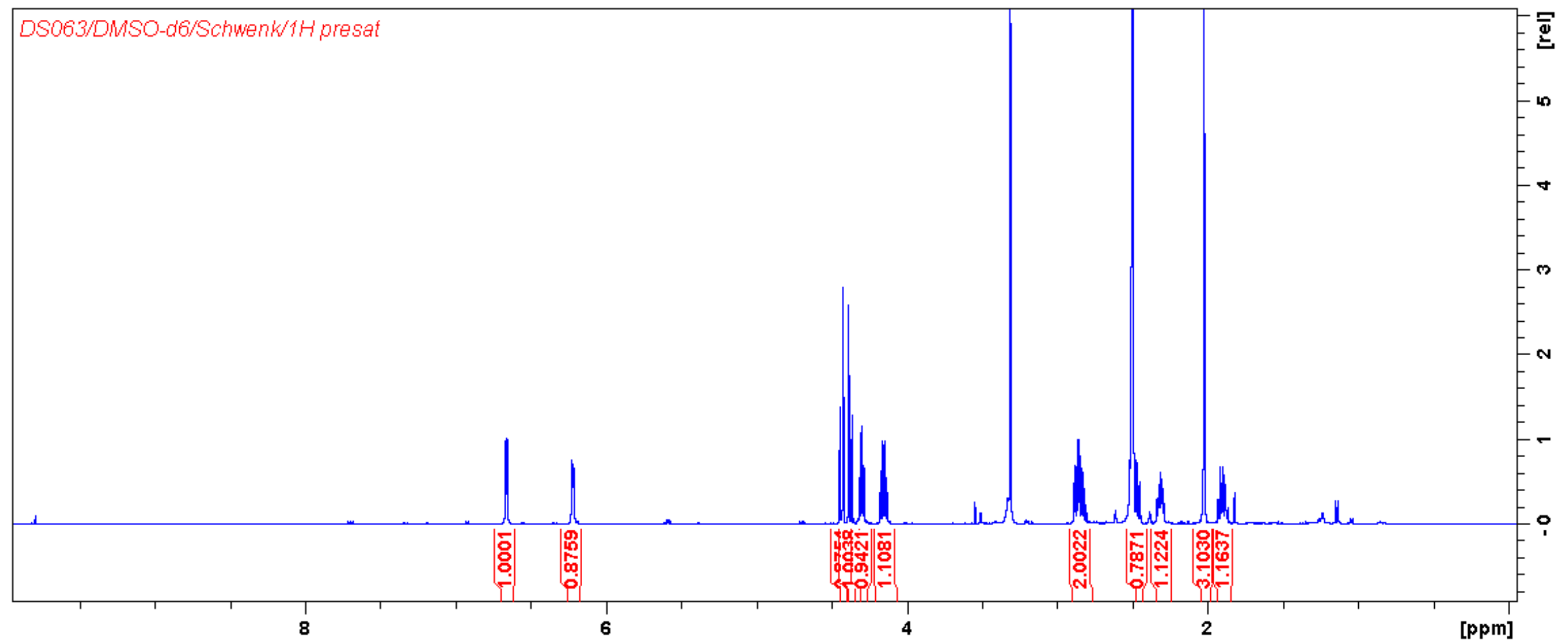

Figure S9. ${ }^{1} \mathrm{H}$ NMR spectrum of vibralactone $\mathrm{S}(4)$. 


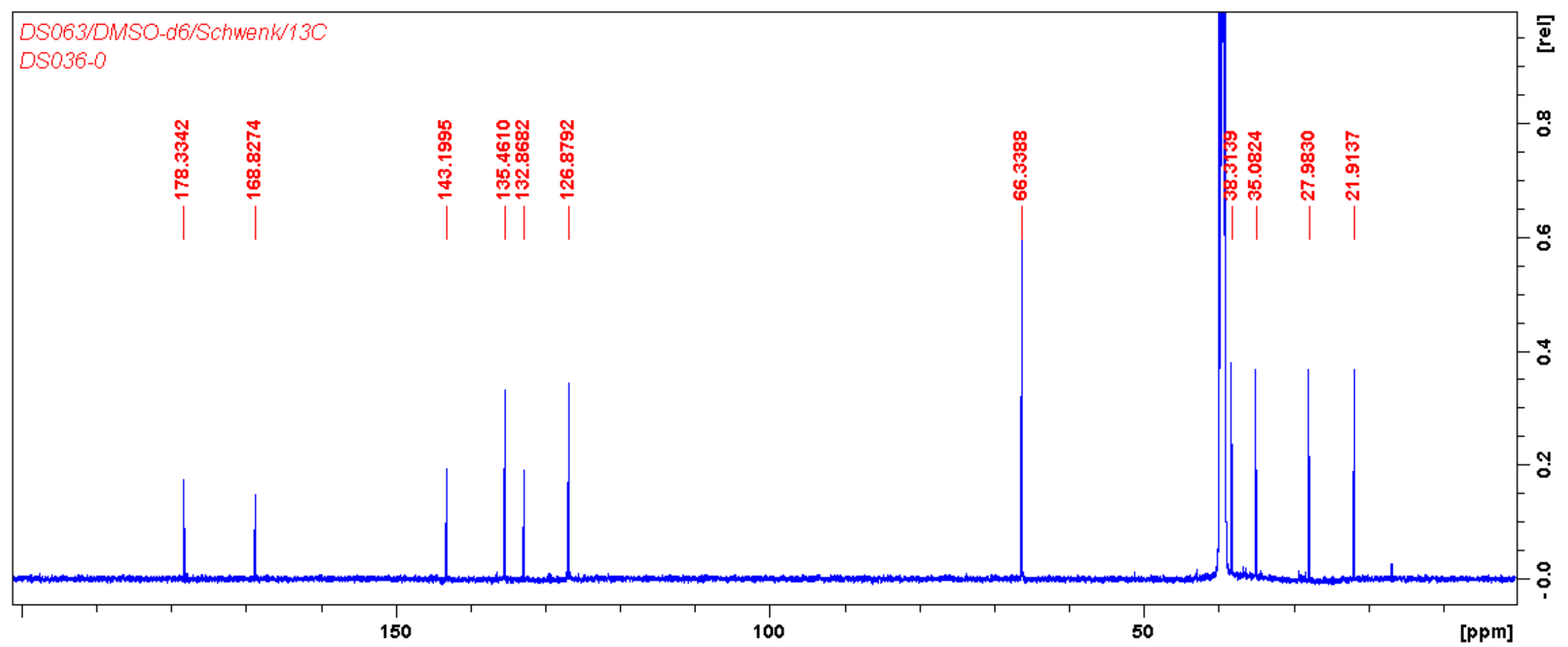

Figure S10. ${ }^{1} \mathrm{H}$-decoupled ${ }^{13} \mathrm{C}$ NMR spectrum of vibralactone $\mathrm{S}(4)$. 


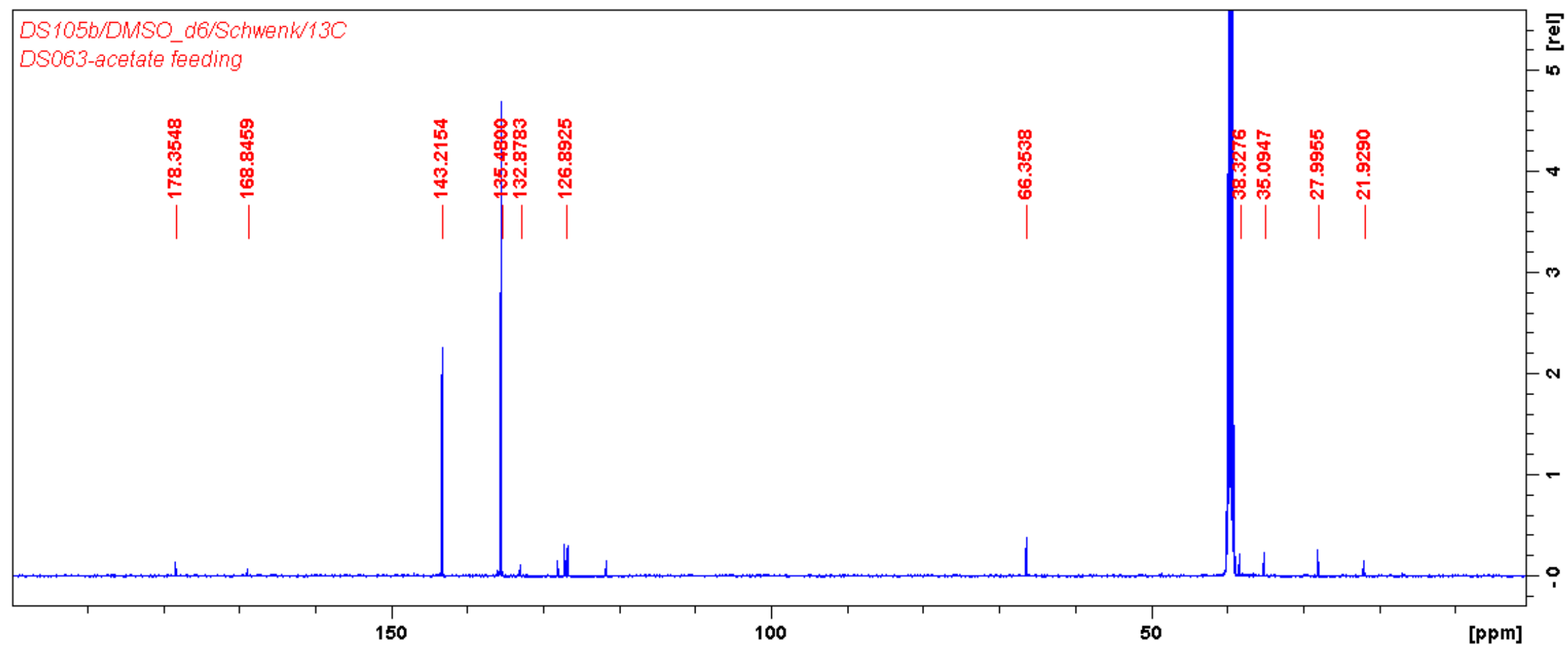

Figure S11. ${ }^{1} \mathrm{H}$-decoupled ${ }^{13} \mathrm{C}$ NMR spectrum of 4, isolated from $\left[1-{ }^{13} \mathrm{C}\right]$ acetate-supplemented culture. 


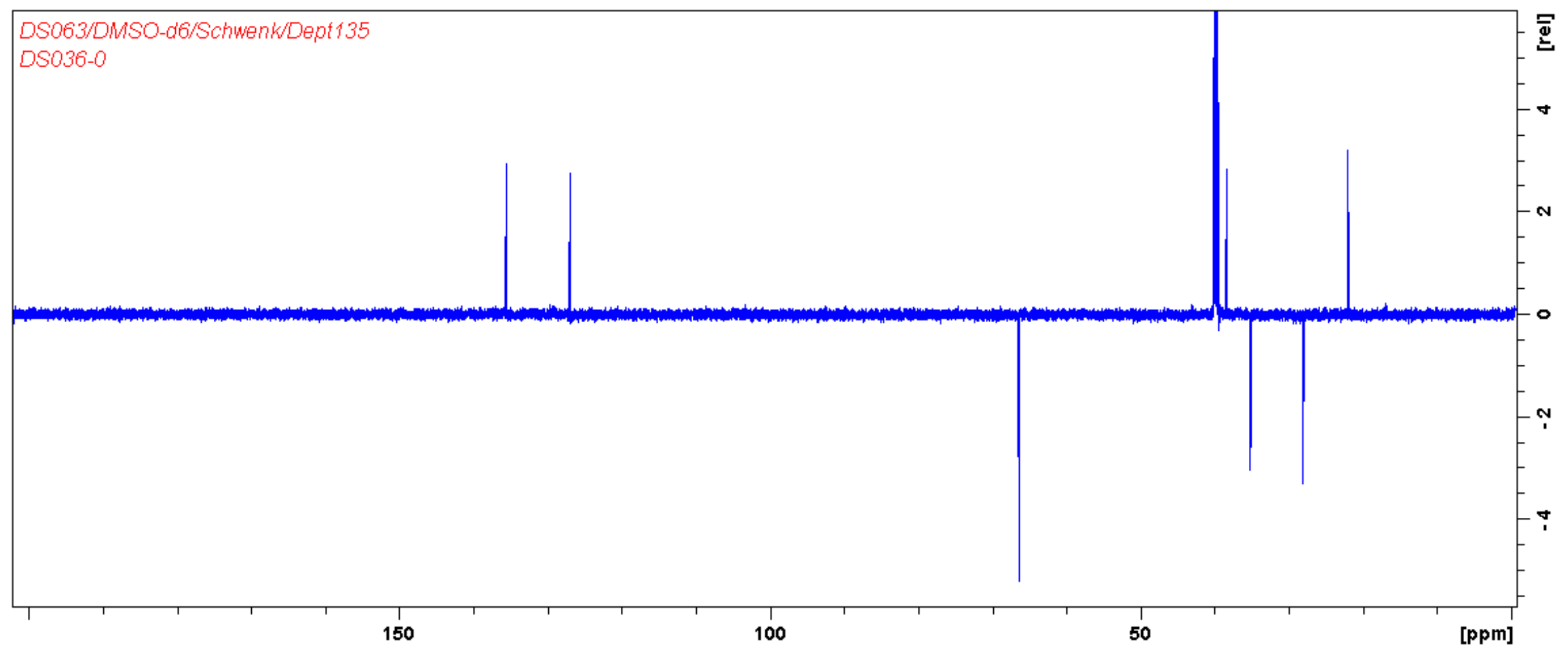

Figure S12. DEPT135 NMR spectrum of vibralactone S (4). 


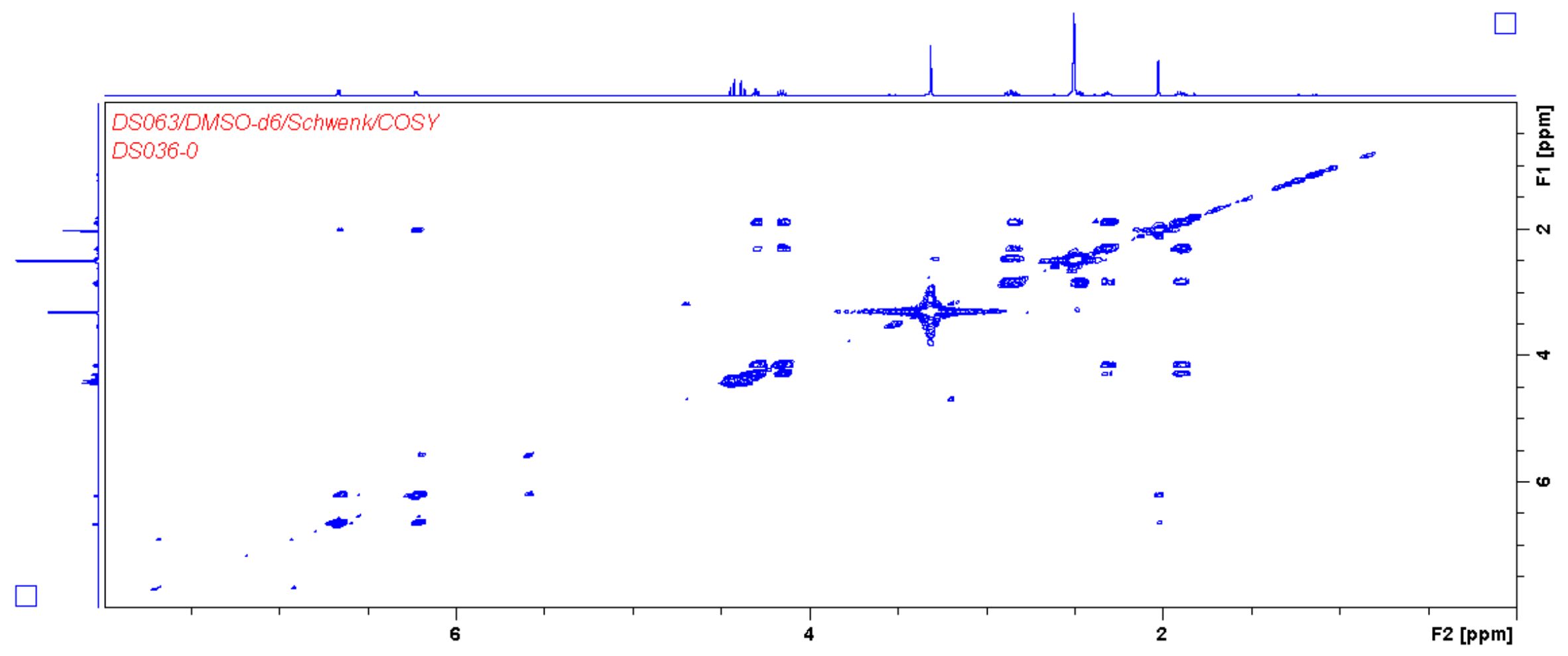

Figure S13. COSY spectrum of vibralactone S (4). 


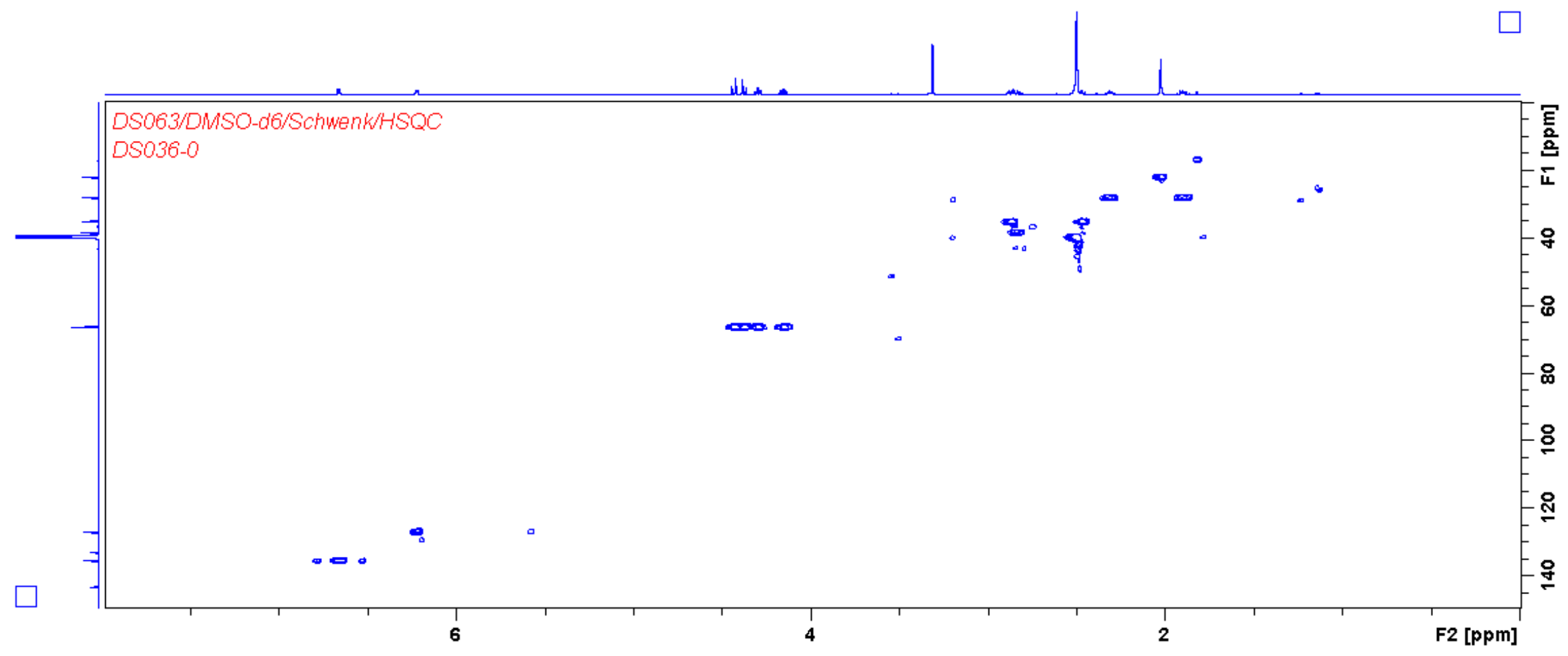

Figure S14. HSQC spectrum of vibralactone S (4). 


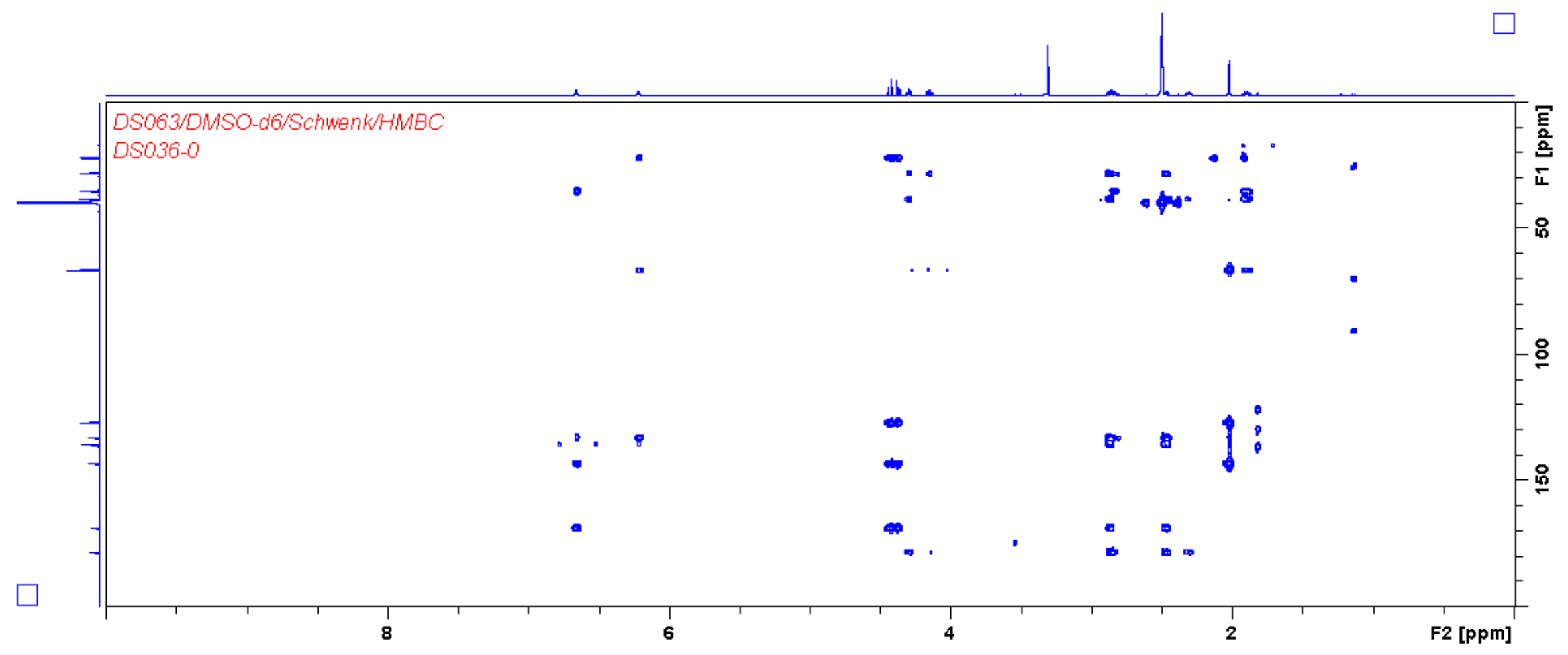

Figure S15. HMBC spectrum of vibralactone S (4). 


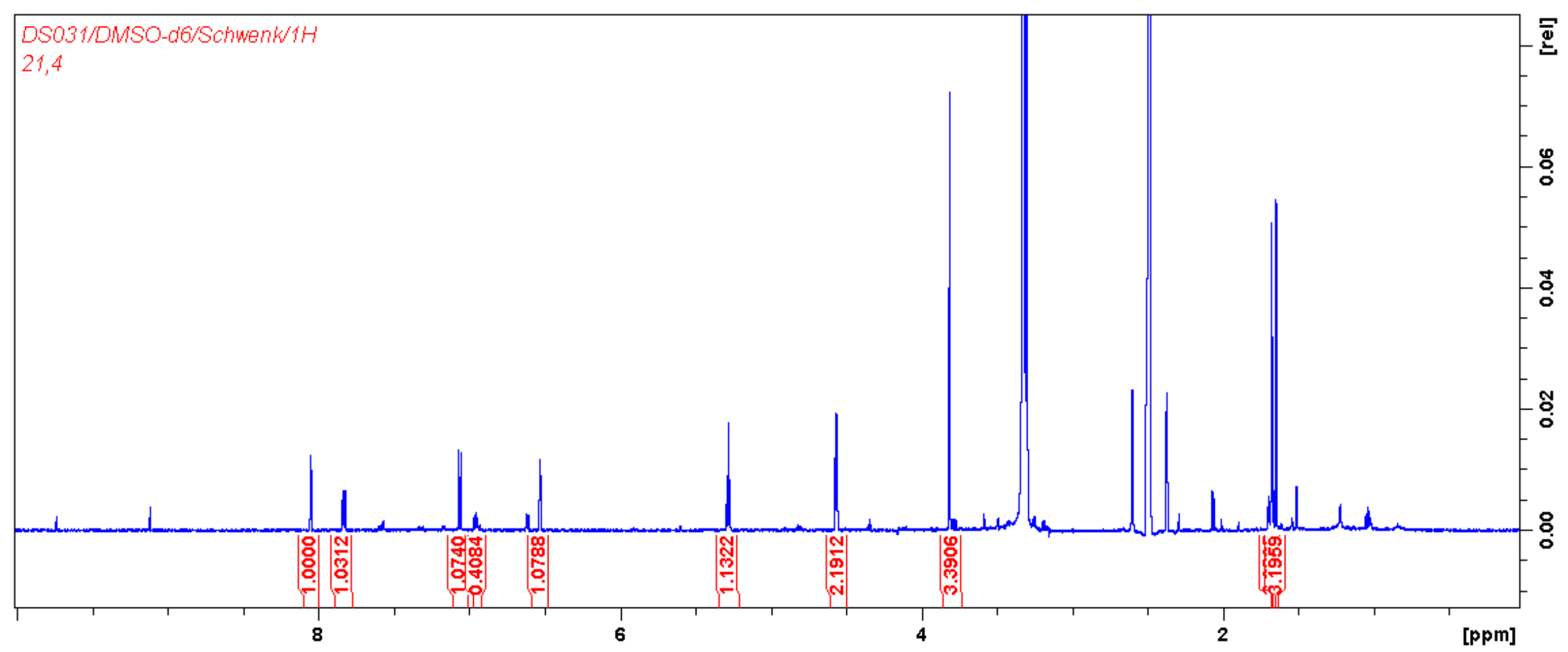

Figure S16. ${ }^{1} \mathrm{H}$ NMR spectrum of methyl seco-fomannoxinate (6). 


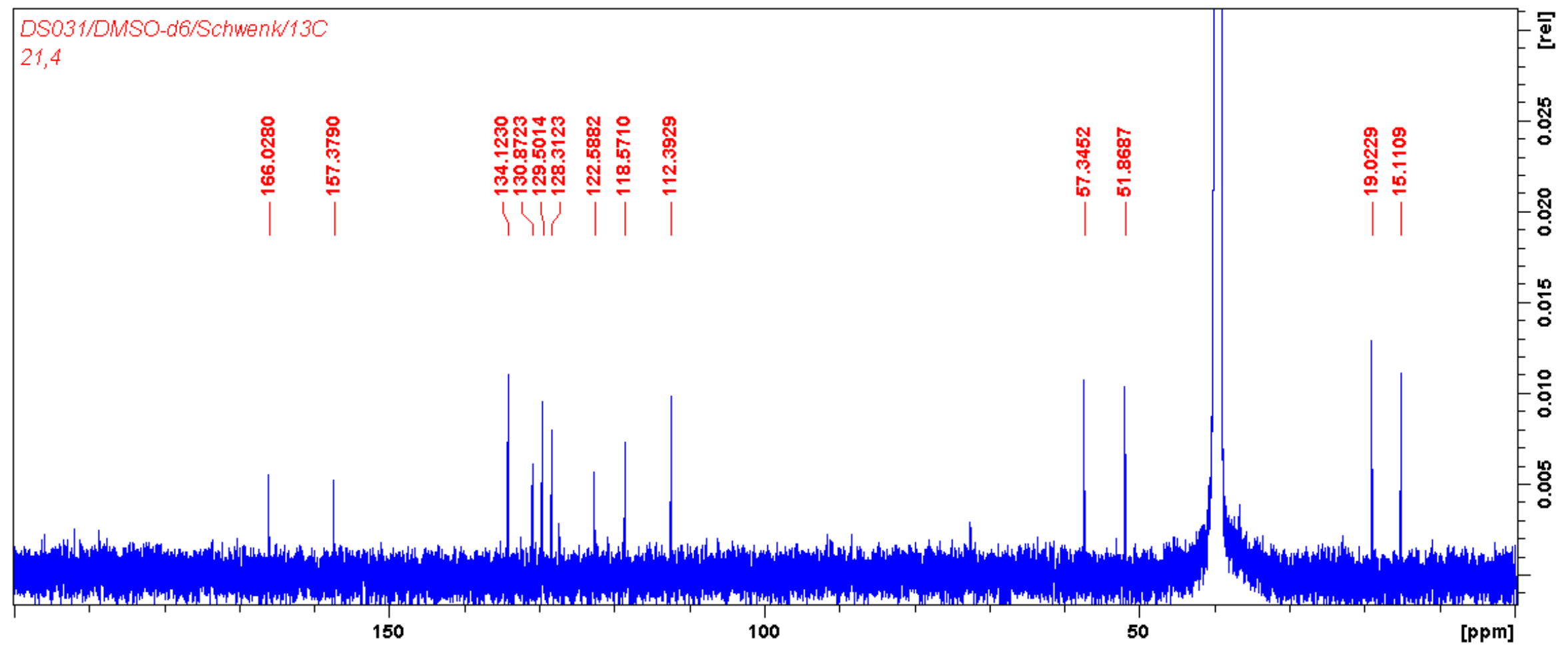

Figure S17. ${ }^{1} \mathrm{H}$-decoupled ${ }^{13} \mathrm{C}$ NMR spectrum of methyl seco-fomannoxinate (6). 


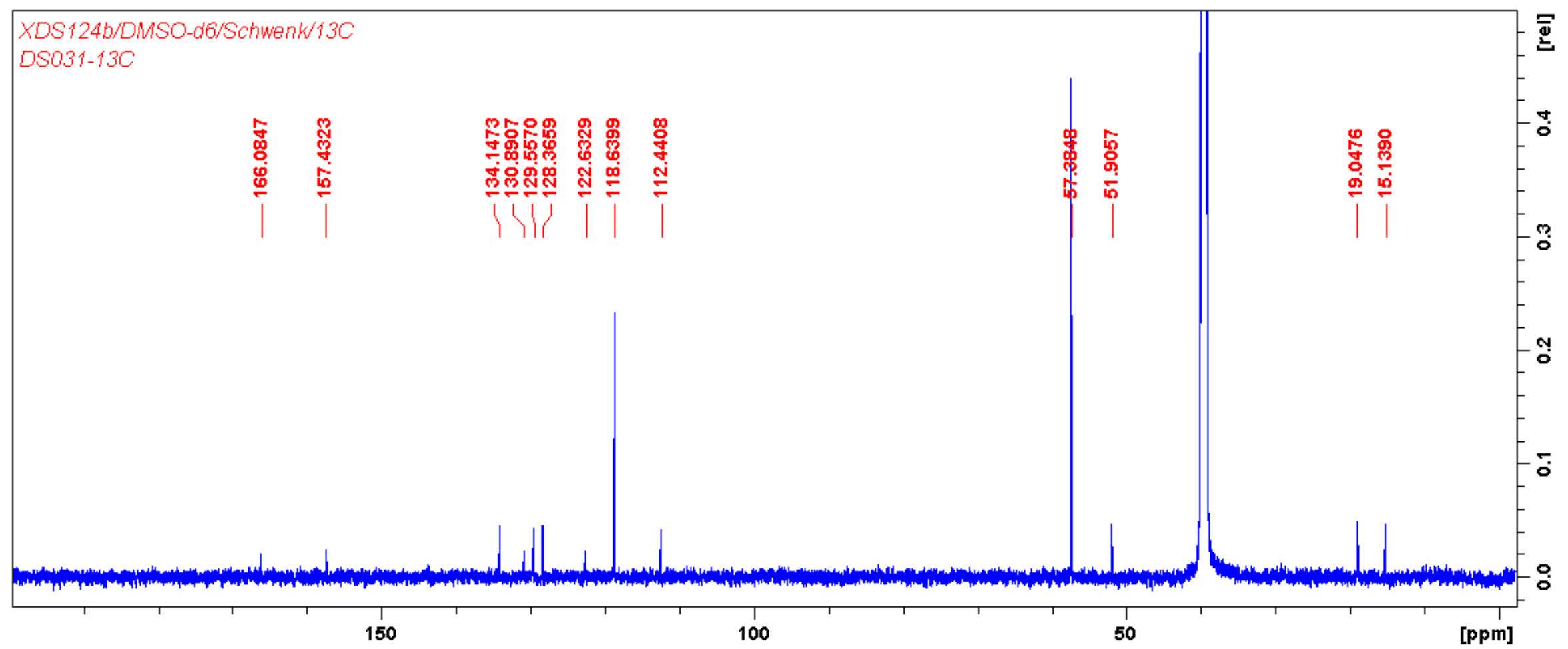

Figure S18. ${ }^{1} \mathrm{H}$-decoupled ${ }^{13} \mathrm{C}$ NMR spectrum of $\mathbf{6}$, isolated from $\left[1-{ }^{13} \mathrm{C}\right]$ acetate-supplemented culture. 


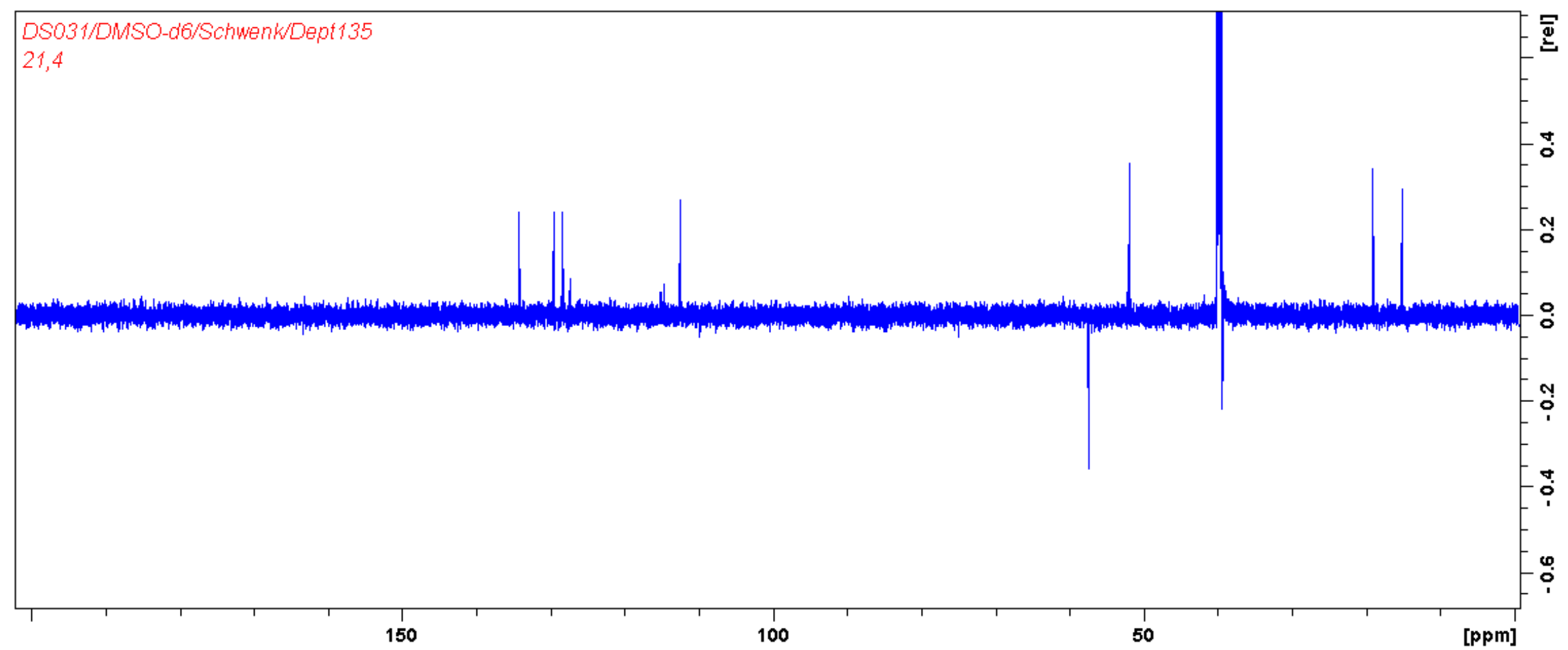

Figure S19. DEPT135 pectrum of methyl seco-fomannoxinate (6). 


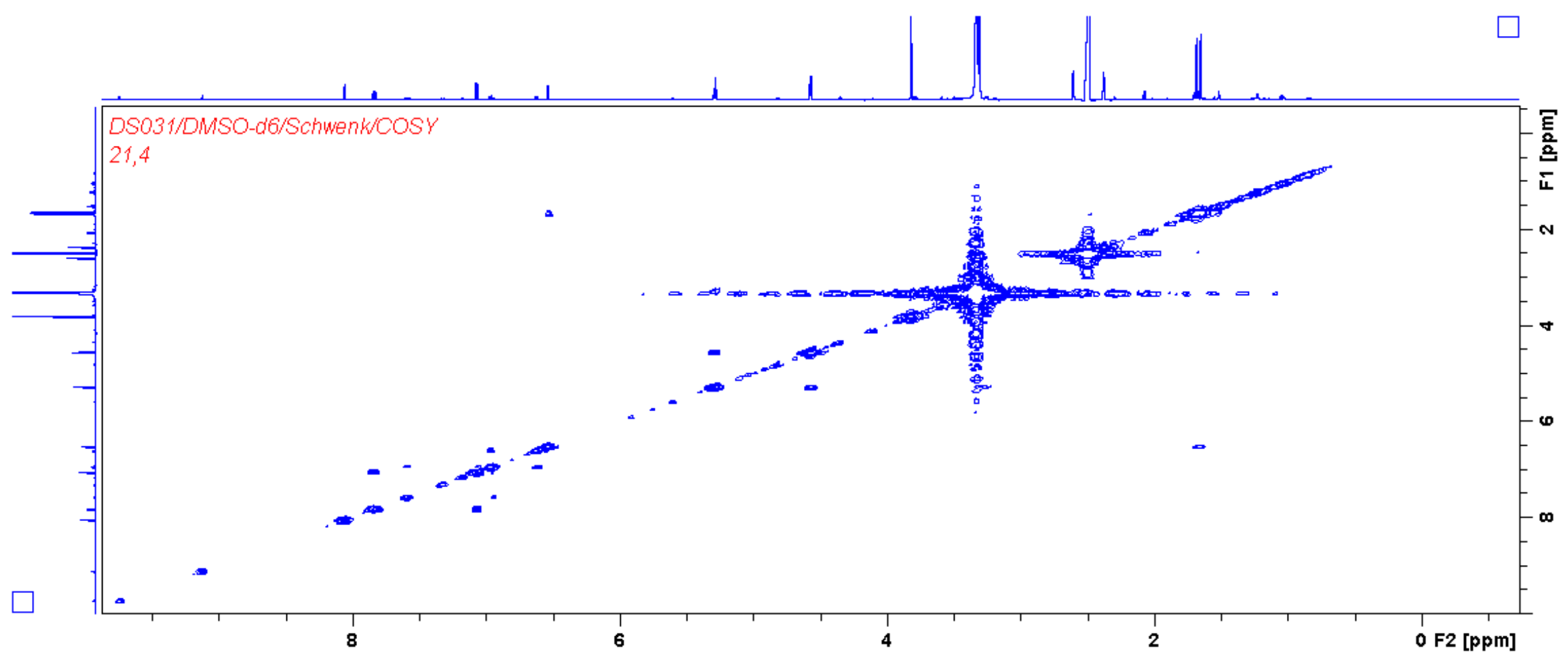

Figure S20. COSY spectrum of methyl seco-fomannoxinate (6). 


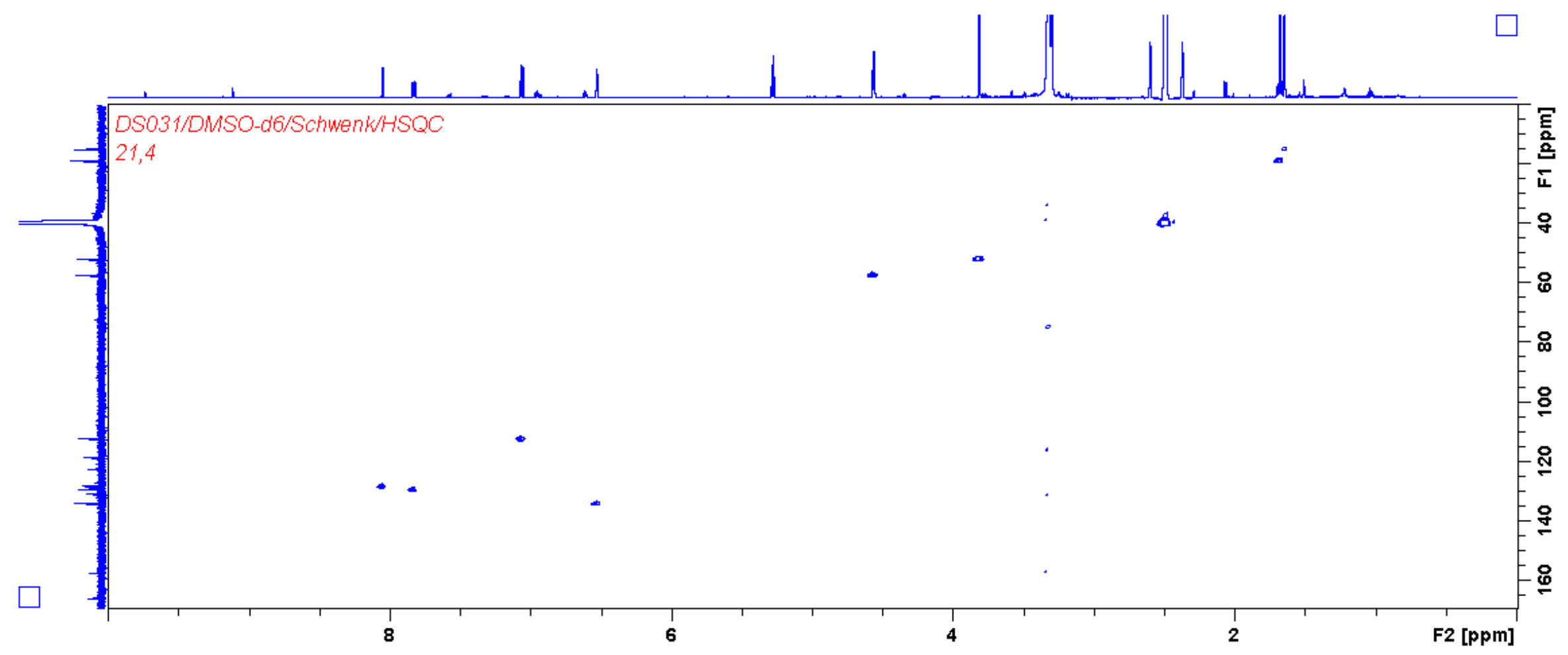

Figure S21. HSQC spectrum of methyl seco-fomannoxinate (6). 


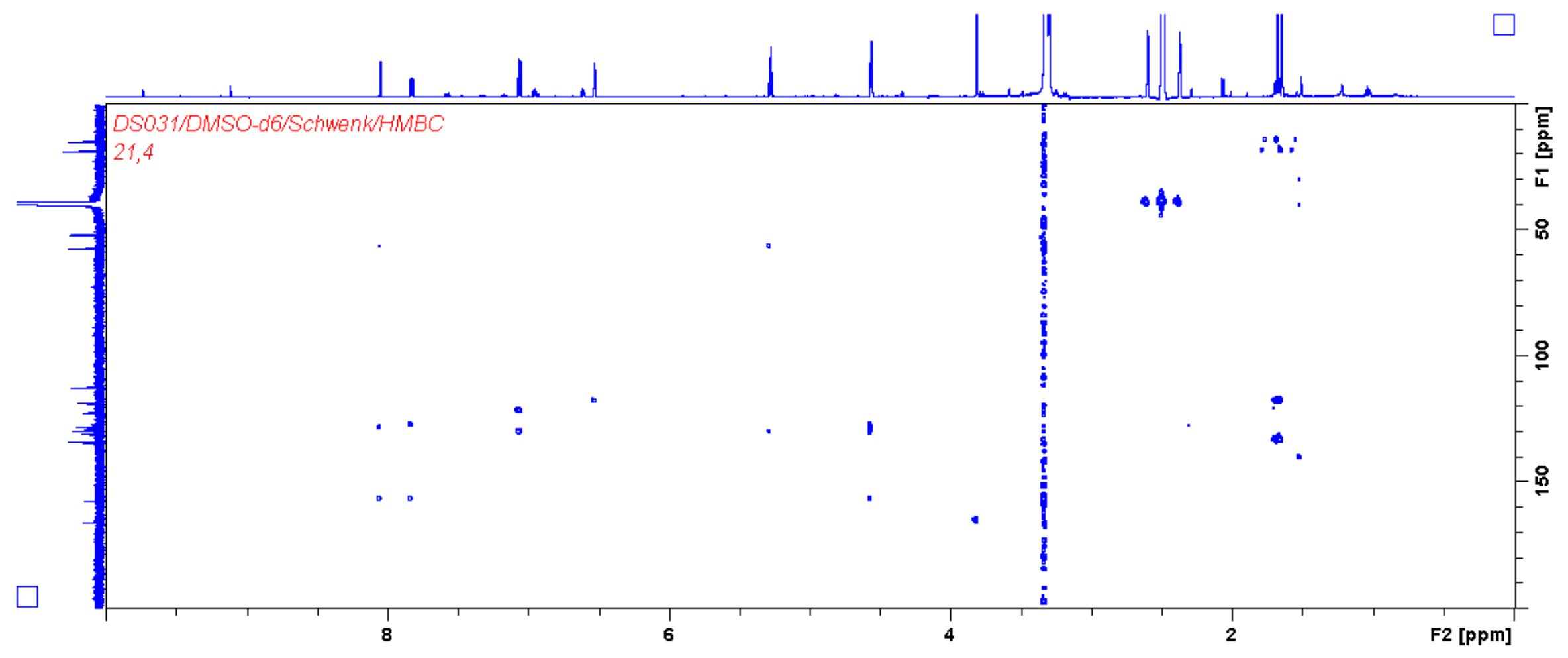

Figure S22. HMBC spectrum of methyl seco-fomannoxinate (6). 


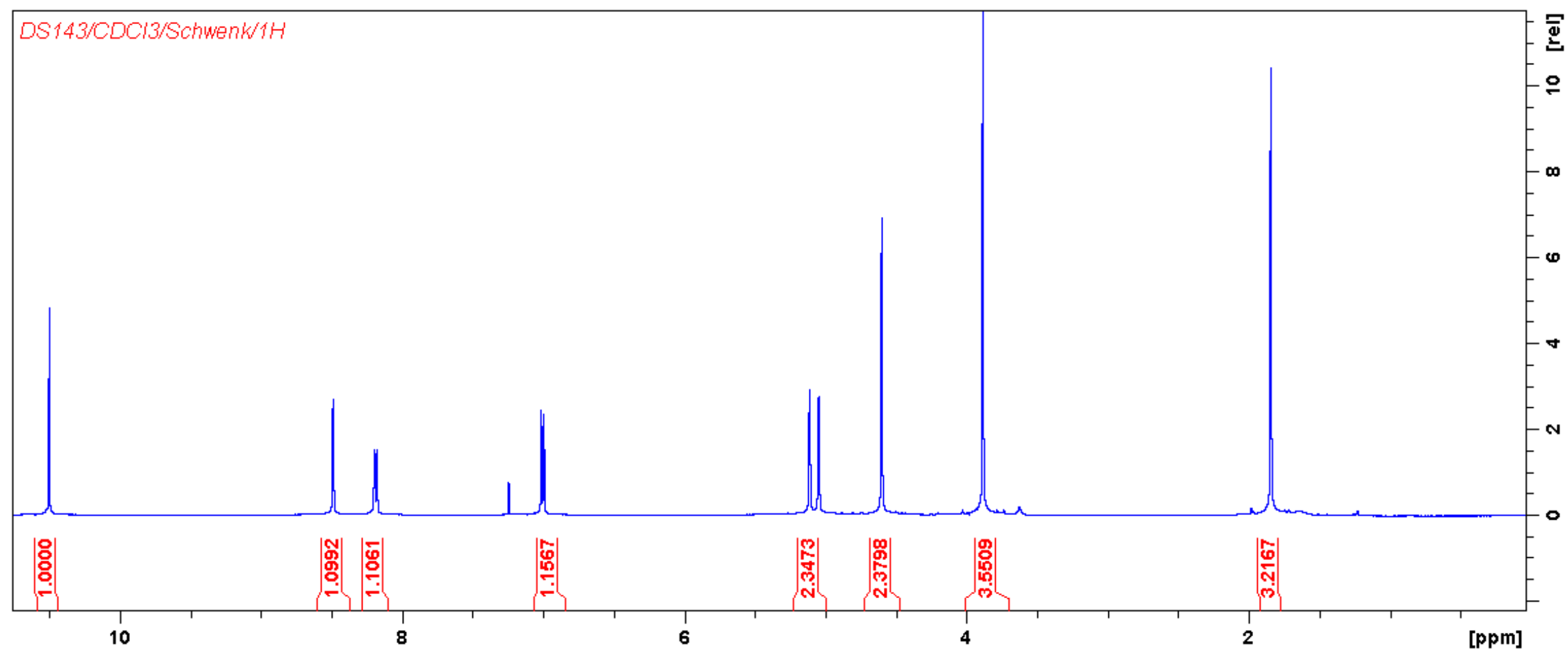

Figure S23. ${ }^{1} \mathrm{H}$ NMR spectrum of synthetic compound 7 . 


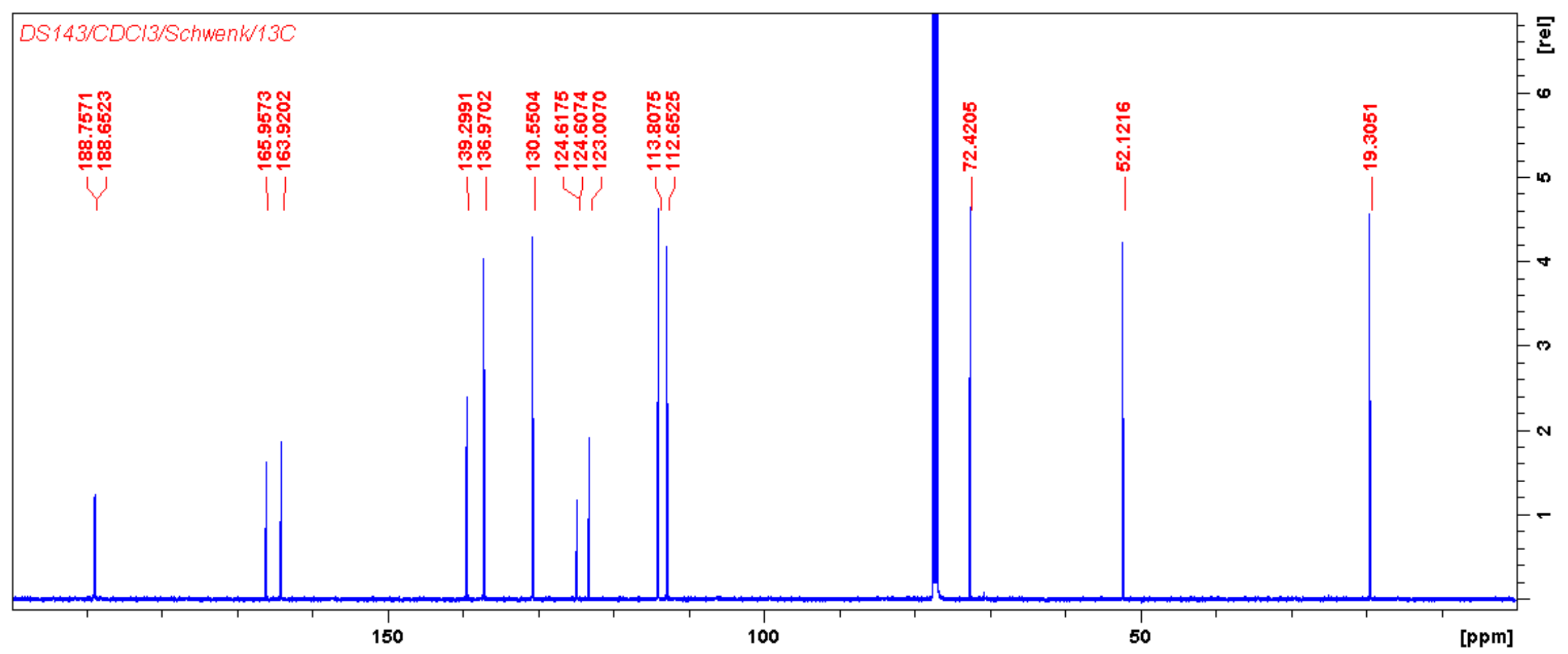

Figure S24. ${ }^{1} \mathrm{H}$-decoupled ${ }^{13} \mathrm{C}$ NMR spectrum of synthetic compound 7 . 


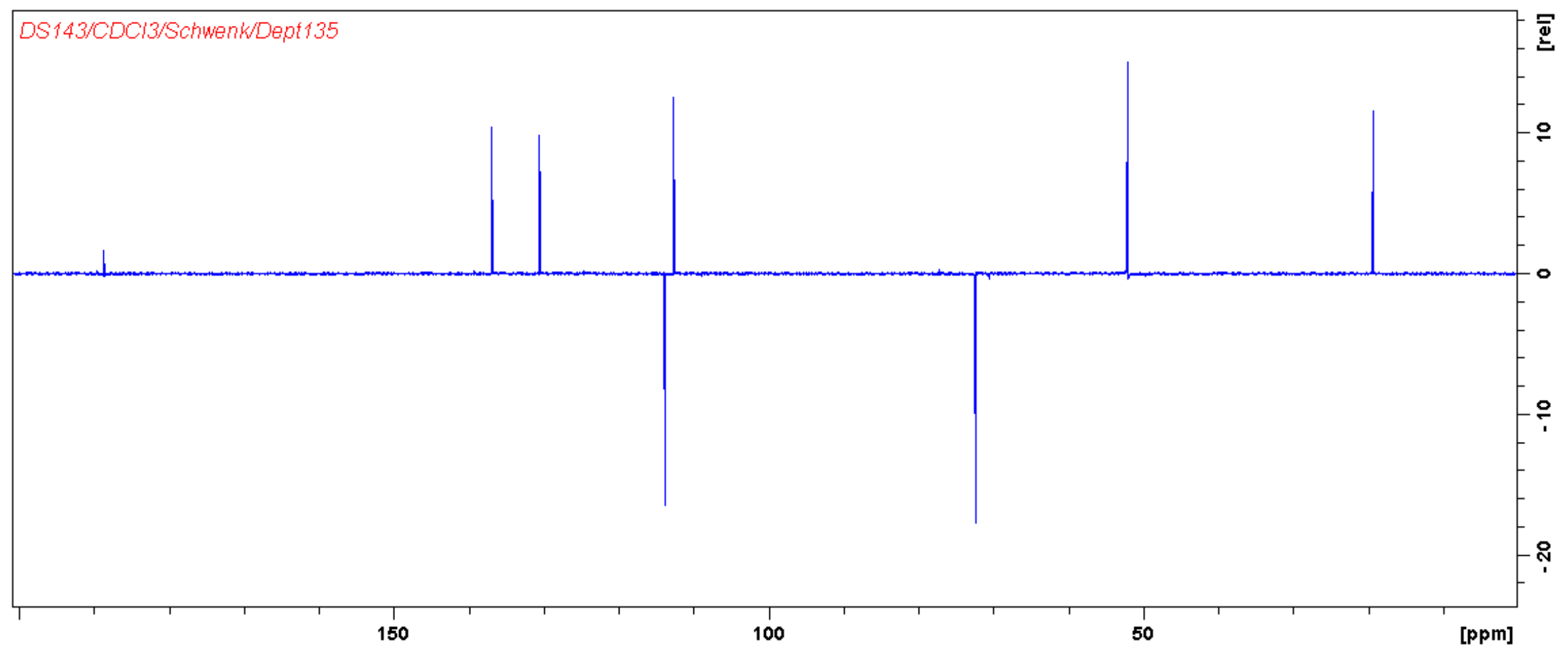

Figure S25. DEPT135 spectrum of synthetic compound 7. 


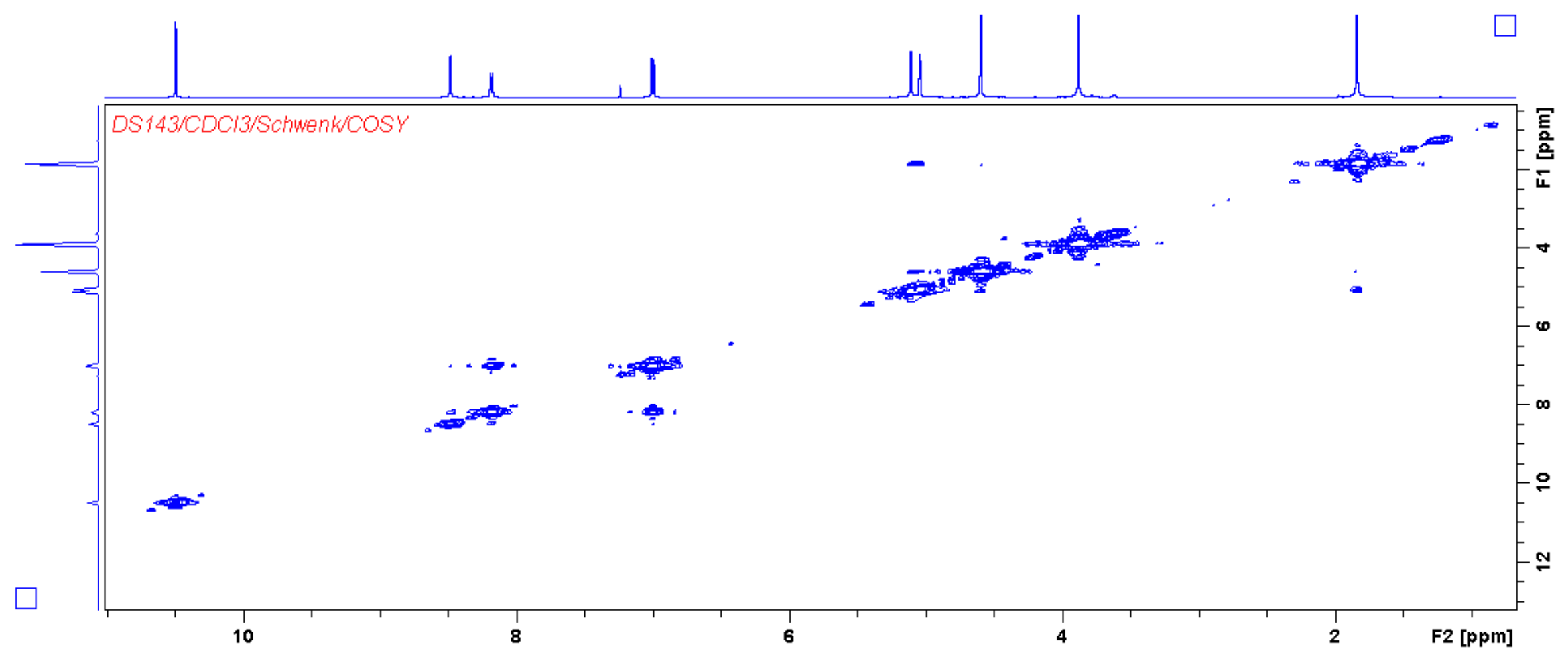

Figure S26. COSY spectrum of synthetic compound 7. 


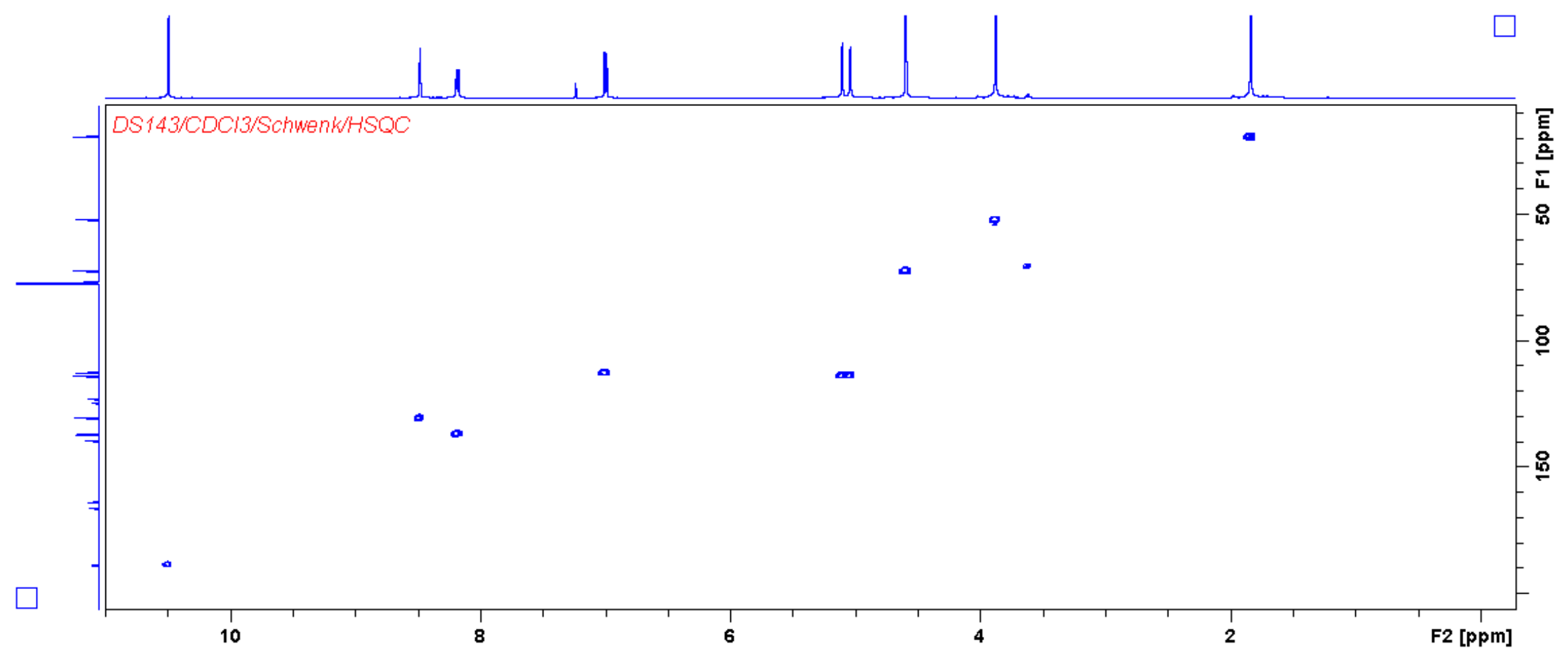

Figure S27. HSQC spectrum of synthetic compound 7. 


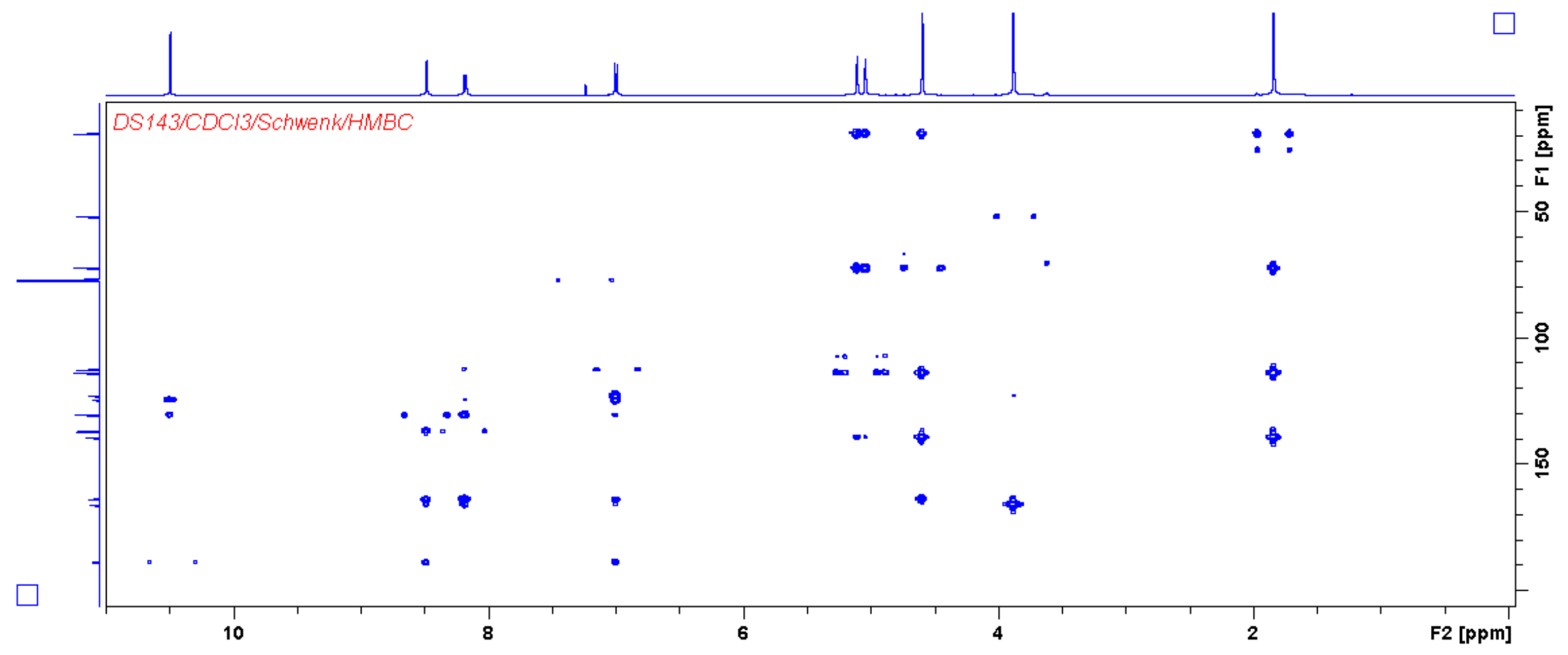

Figure S28. HMBC spectrum of synthetic compound 7. 


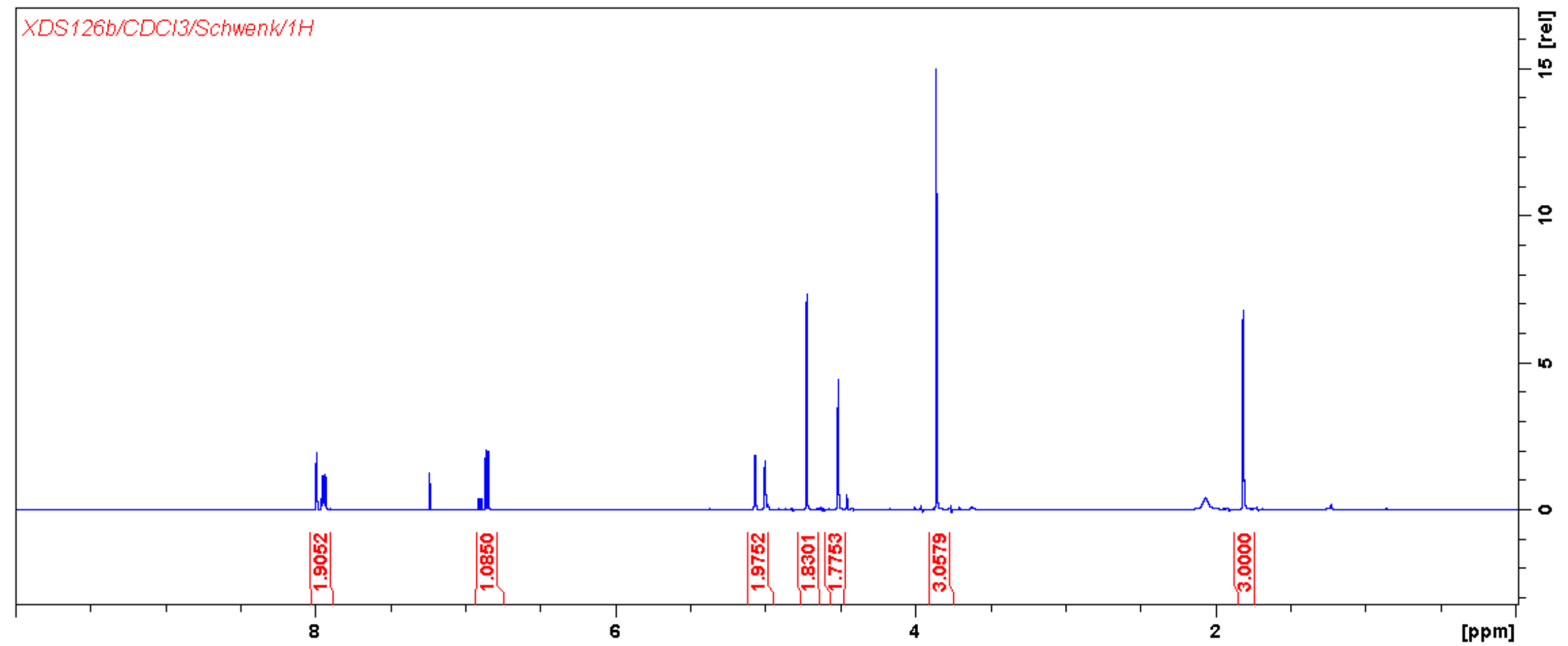

Figure S29. ${ }^{1} \mathrm{H}$ NMR spectrum of synthetic compound 8. 


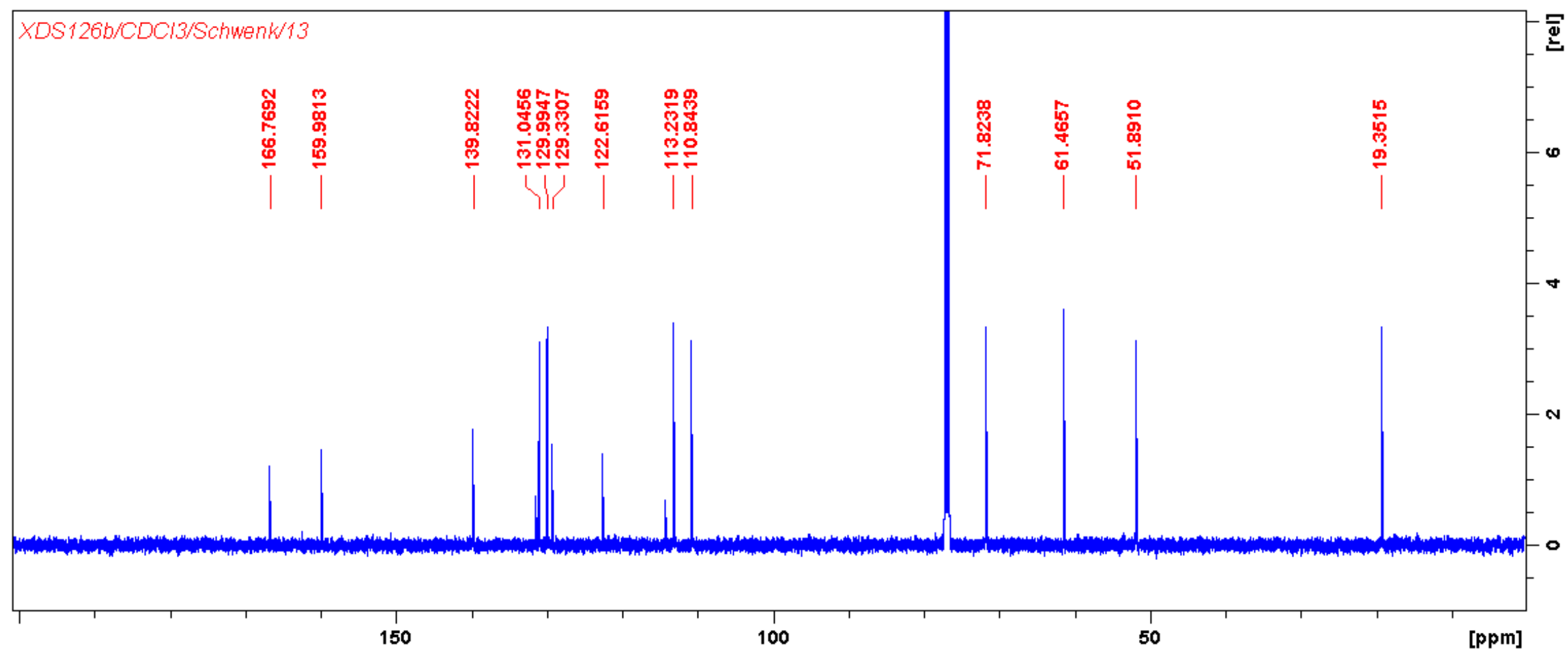

Figure S30. ${ }^{1} \mathrm{H}$-decoupled ${ }^{13} \mathrm{C}$ NMR spectrum of synthetic compound 8 . 


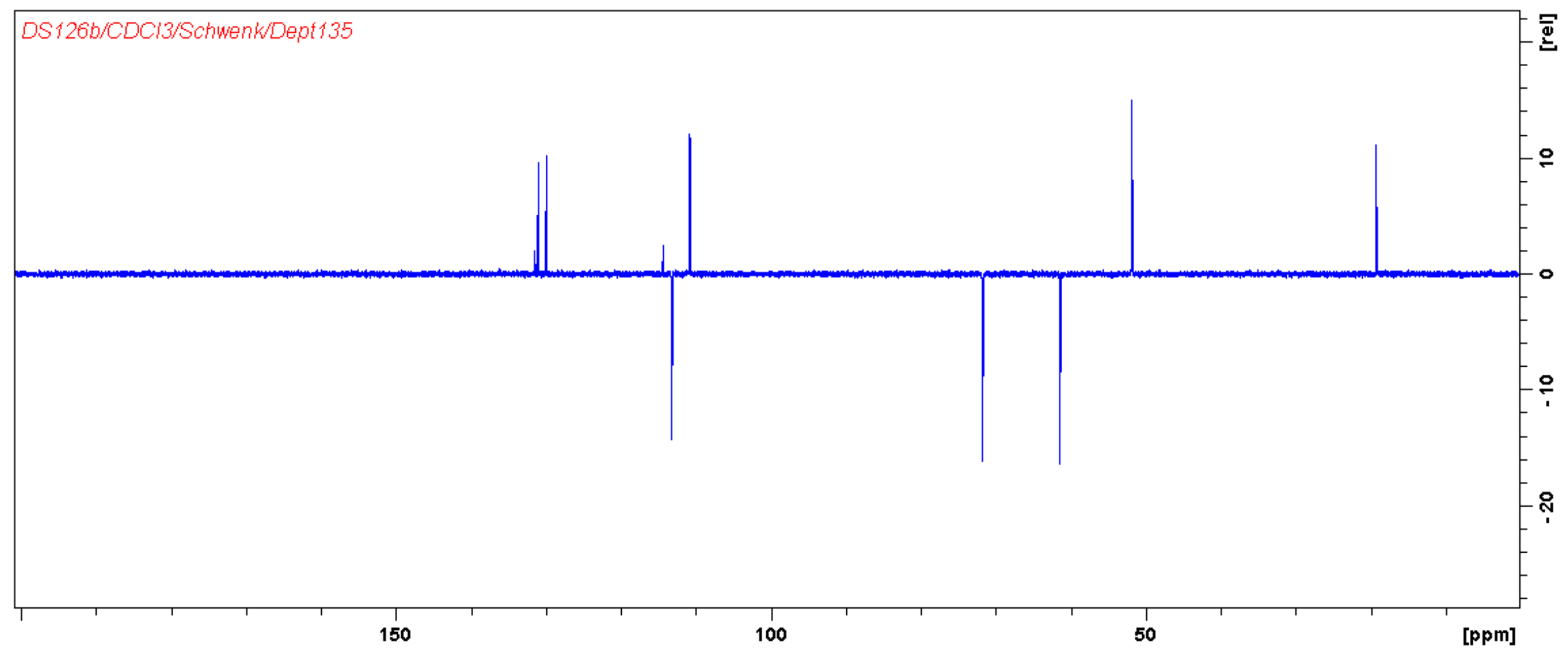

Figure S31. DEPT135 spectrum of synthetic compound 8. 


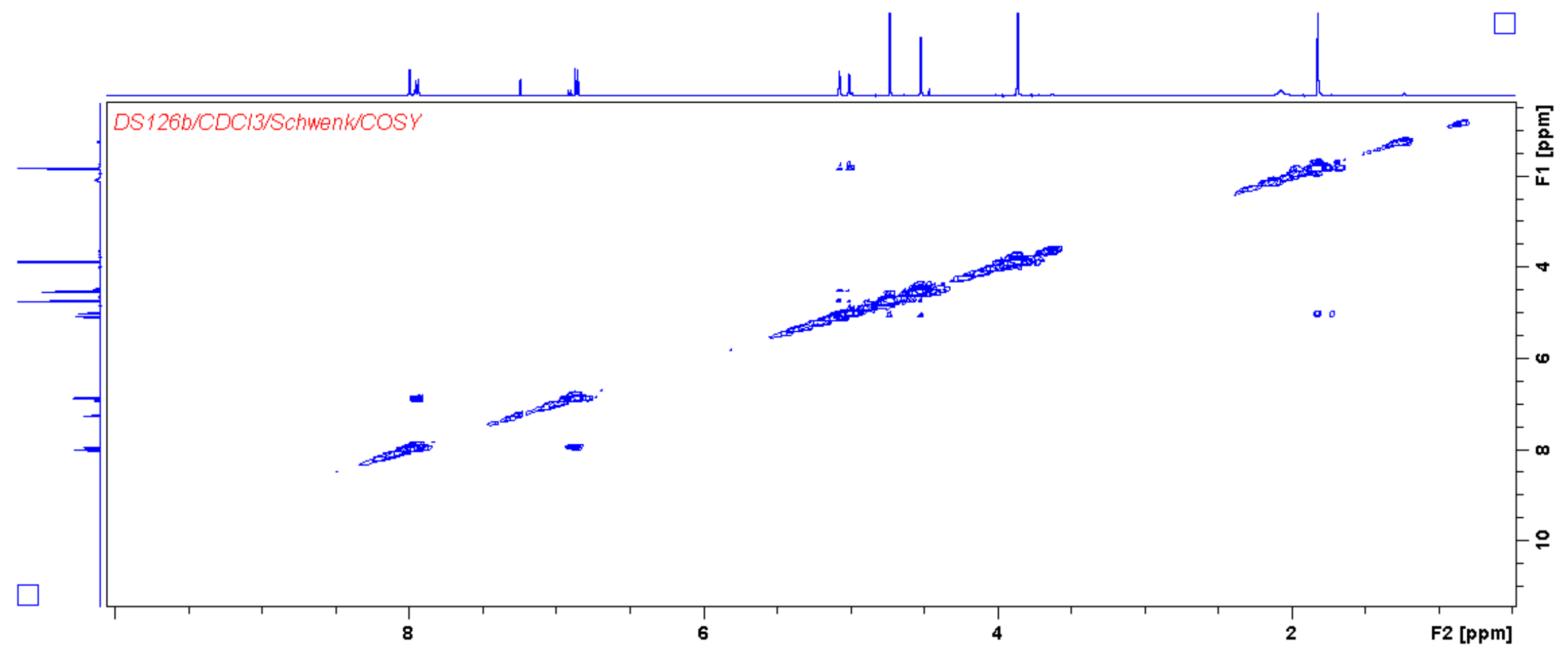

Figure S32. COSY spectrum of synthetic compound 8. 


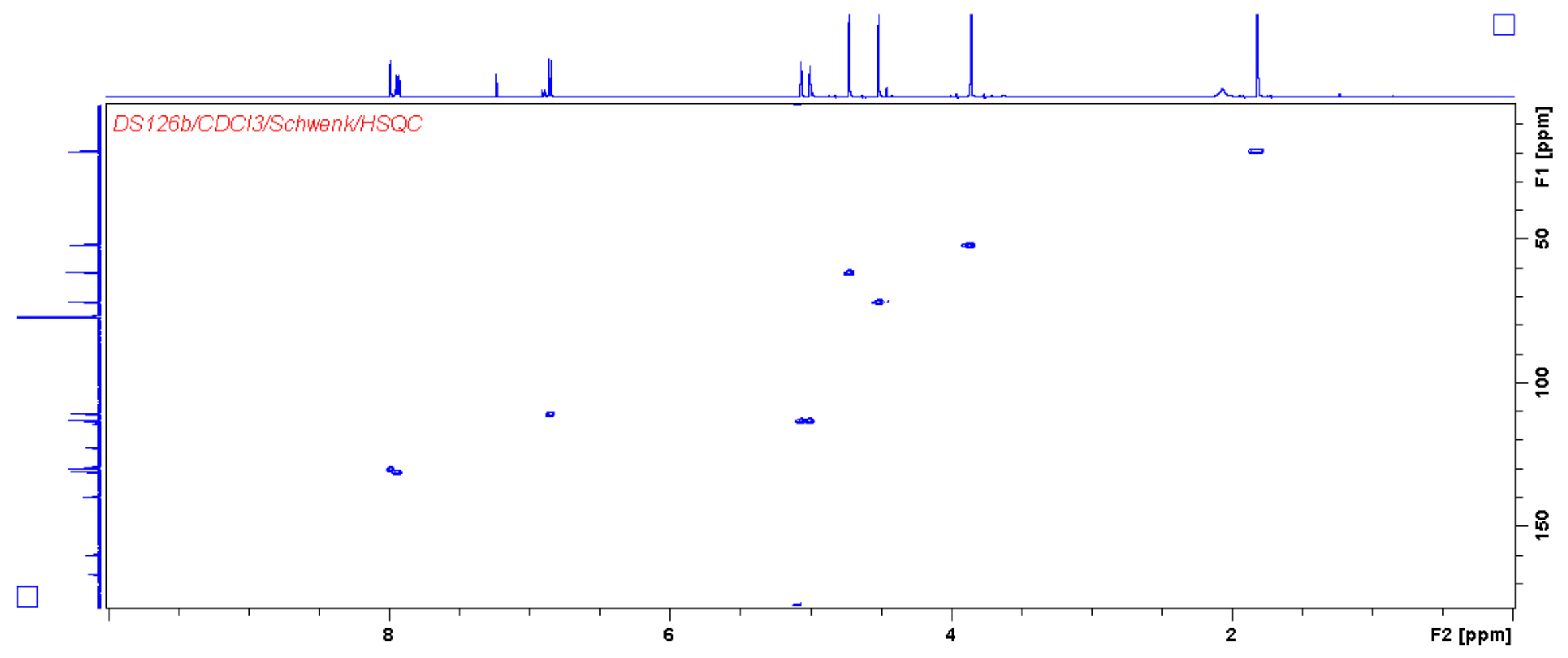

Figure S33. HSQC spectrum of synthetic compound 8. 


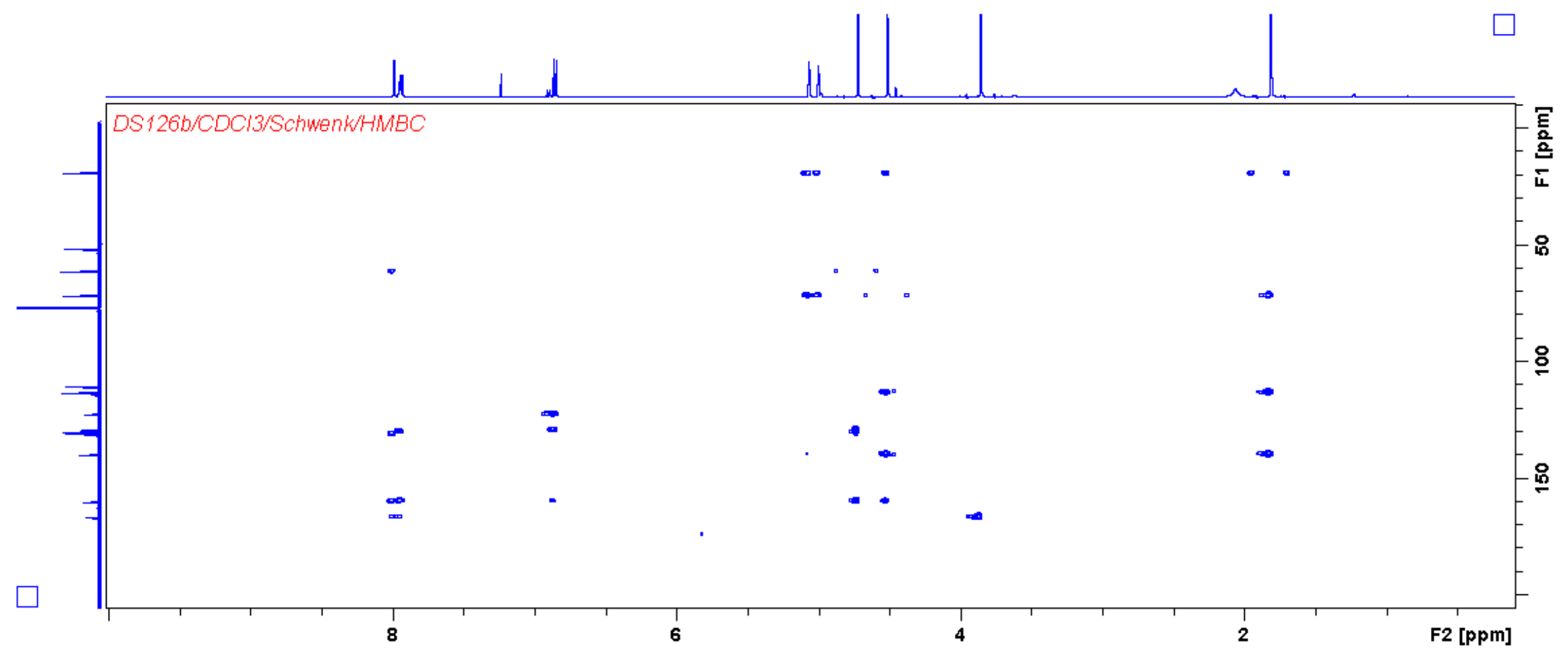

Figure S34. HMBC spectrum of synthetic compound 8. 


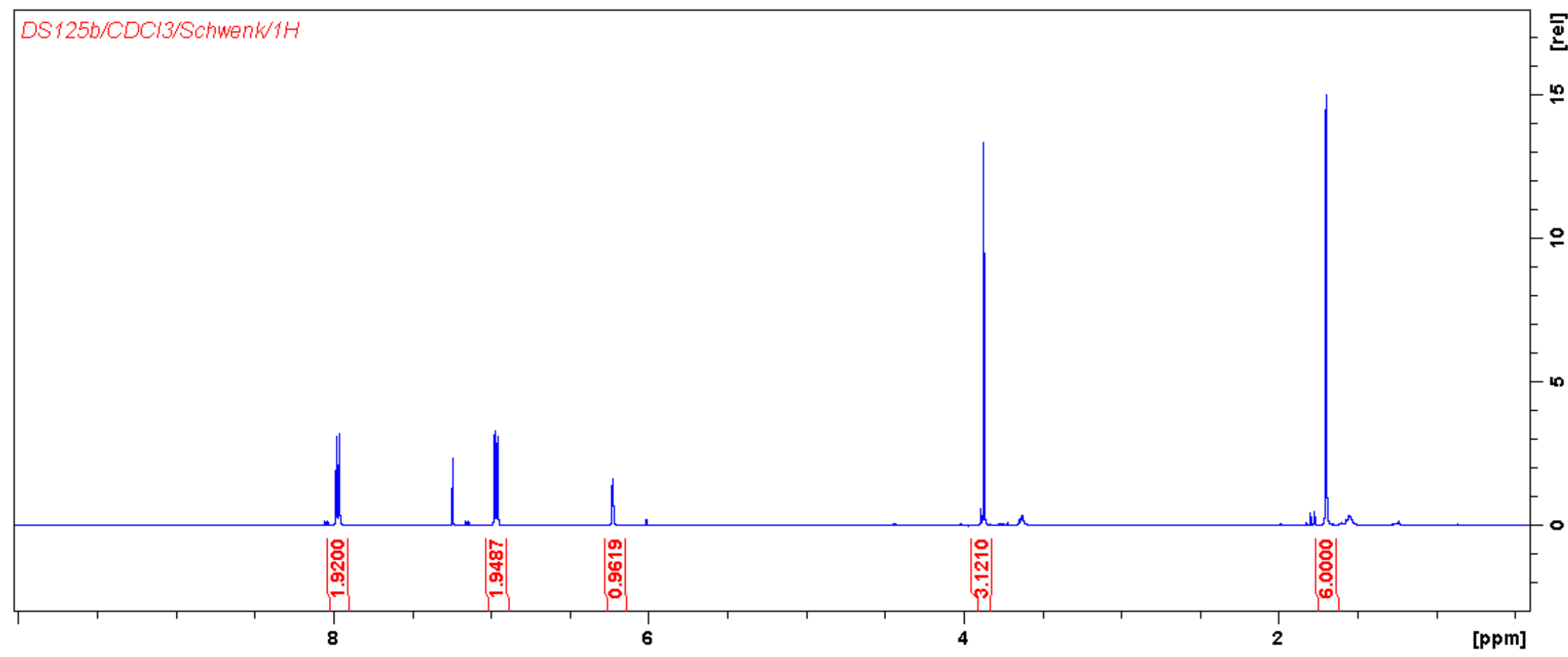

Figure S35. ${ }^{1} \mathrm{H}$ NMR spectrum of synthetic compound 9. 


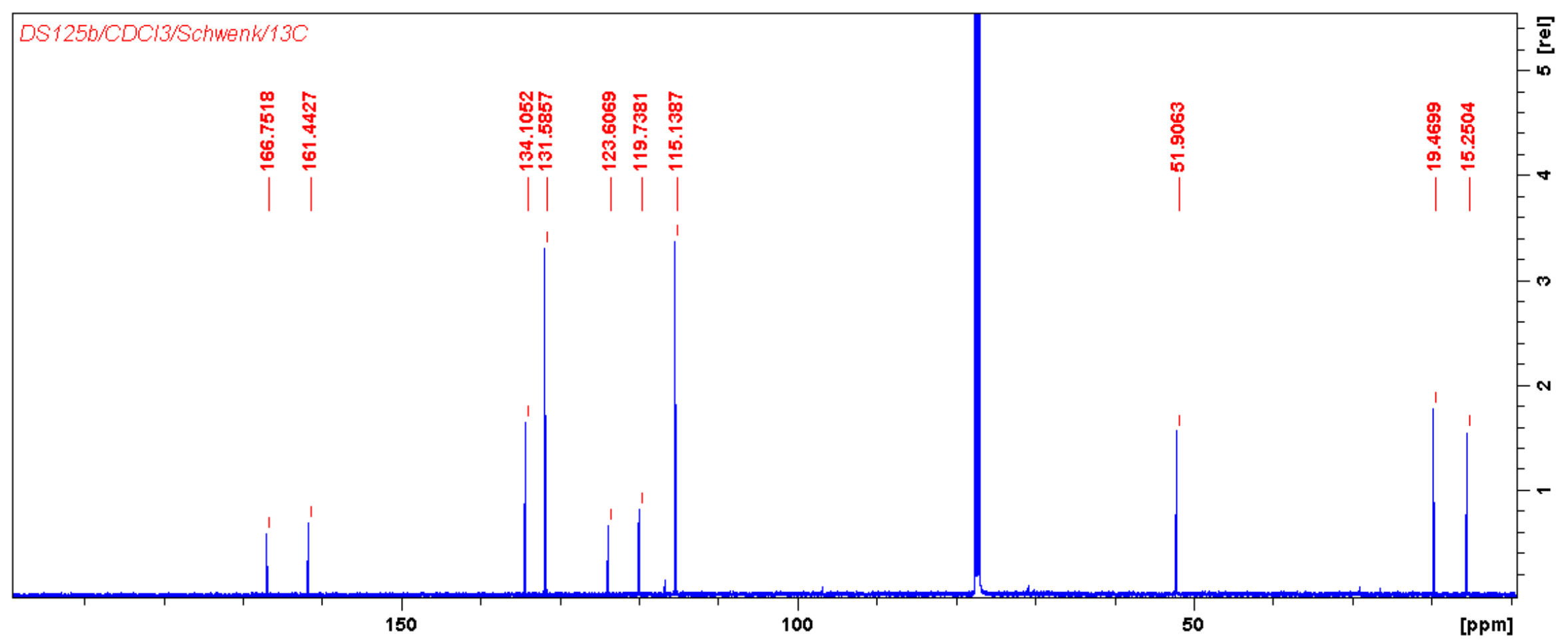

Figure S36. ${ }^{1} \mathrm{H}$-decoupled ${ }^{13} \mathrm{C}$ NMR spectrum of synthetic compound $\mathbf{9}$. 


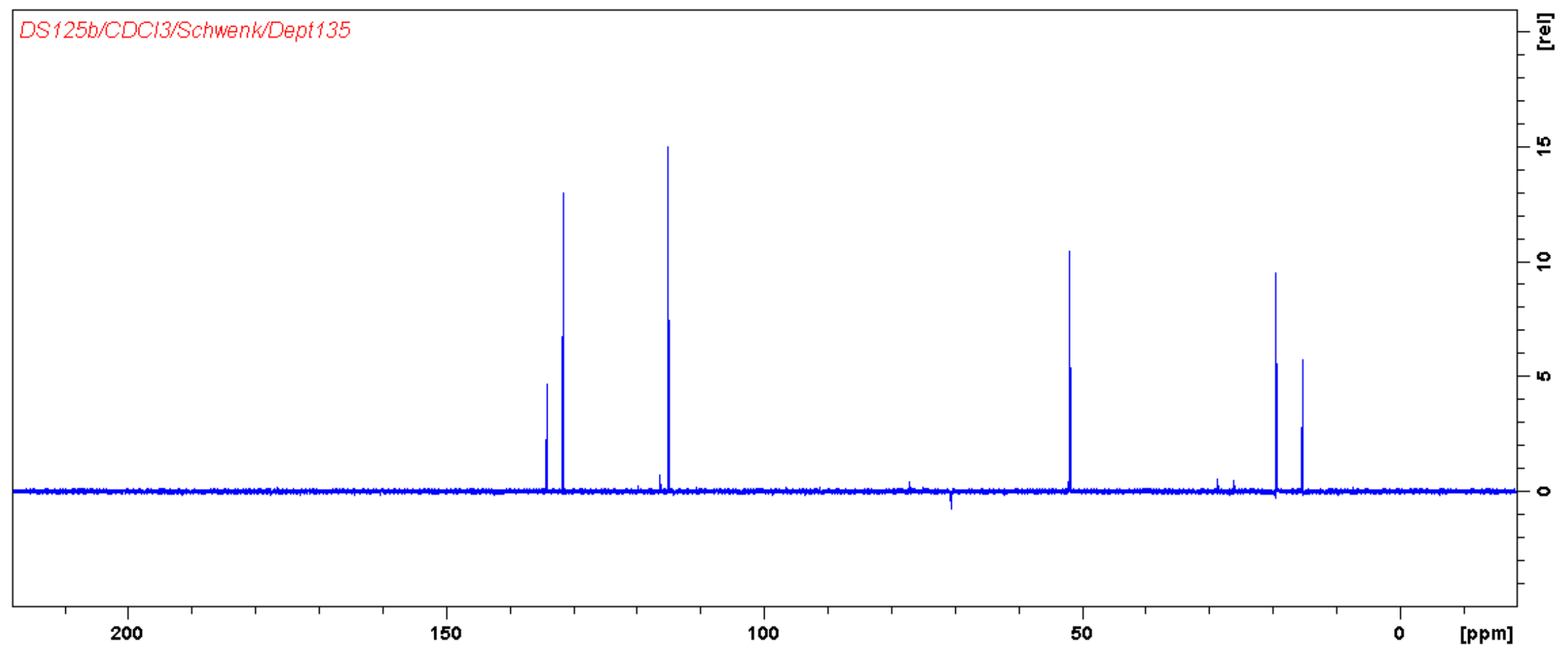

Figure S37. DEPT135 spectrum of synthetic compound 9. 


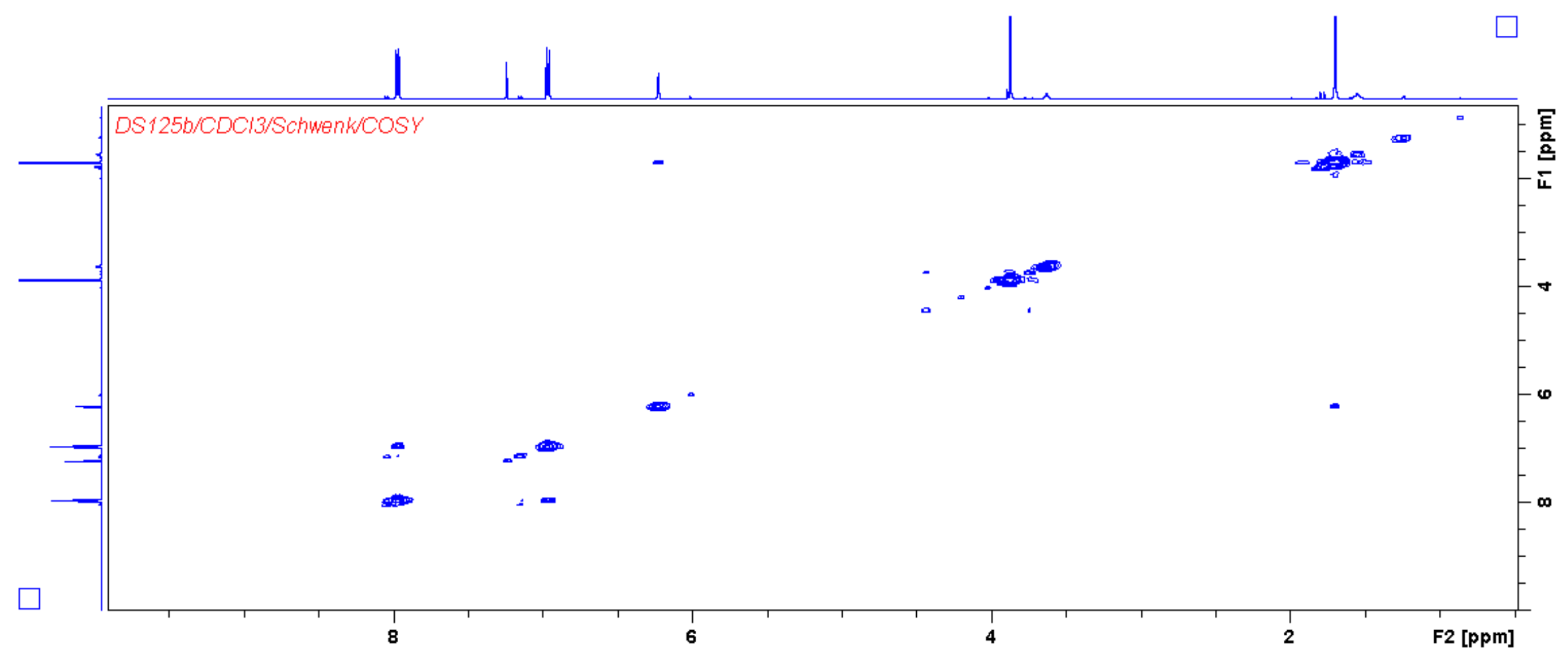

Figure S38. COSY spectrum of synthetic compound $\mathbf{9}$. 


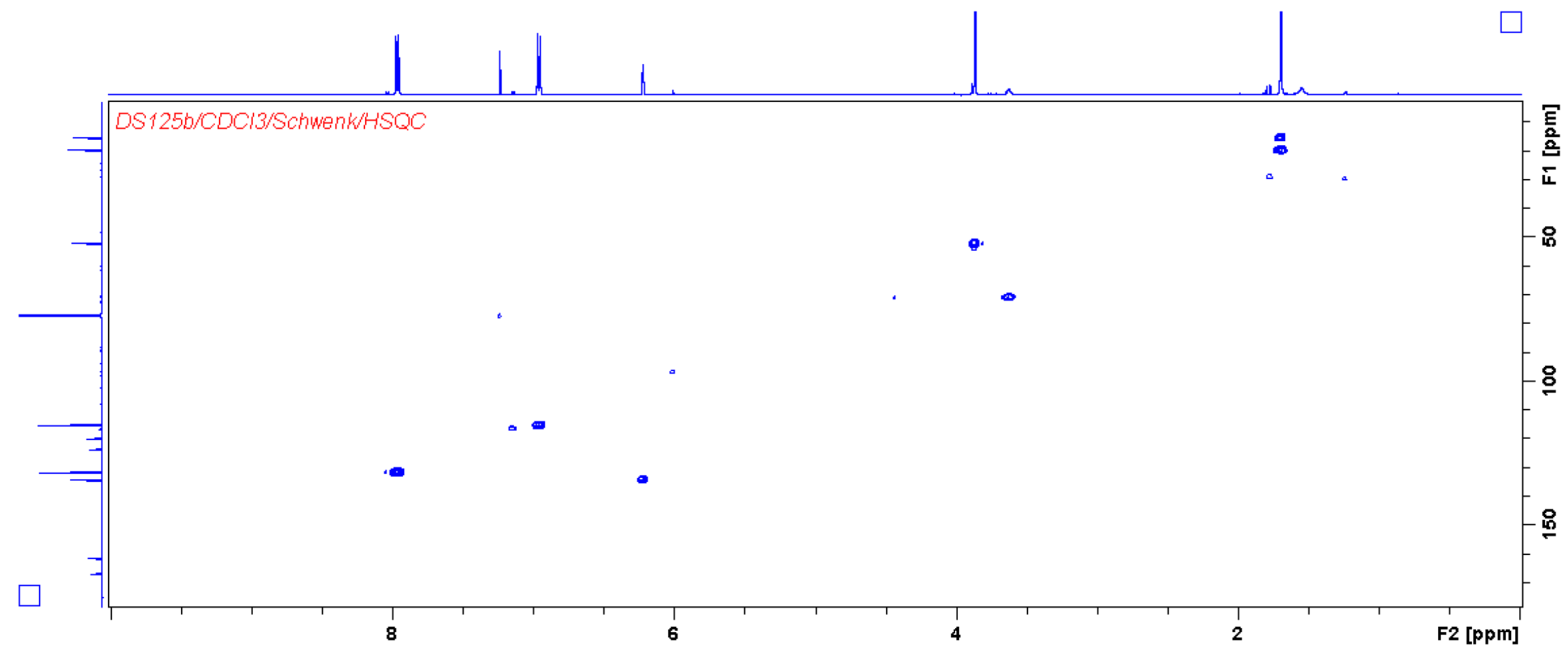

Figure S39. HSQC spectrum of synthetic compound 9. 


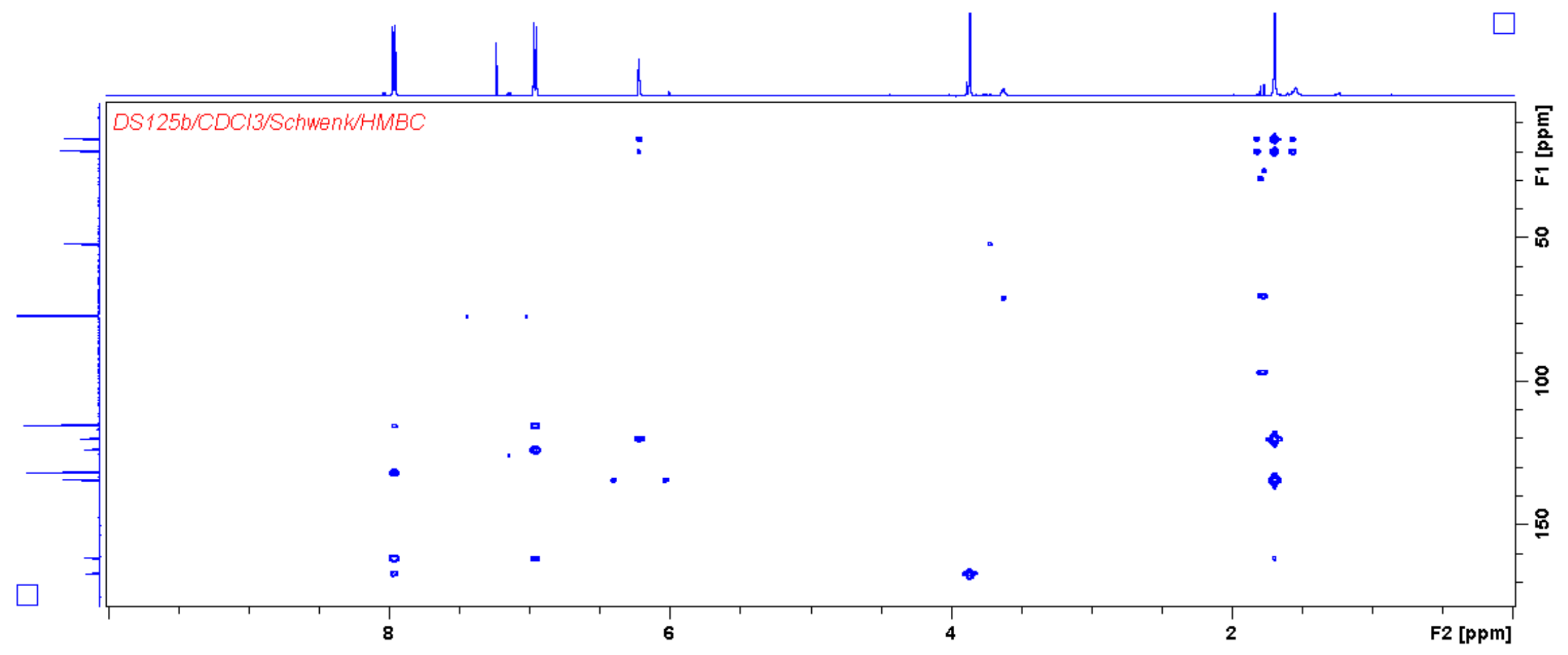

Figure S40. HMBC spectrum of synthetic compound 9 . 